\title{
New a posteriori error estimates of mixed finite element methods for quadratic optimal control problems governed by semilinear parabolic equations with integral constraint
}

\section{Zuliang Lu ${ }^{1,2^{*}}$, Shaohong Du ${ }^{3}$ and Yuelong Tang ${ }^{4}$}

${ }^{*}$ Correspondence:

zulianglux@126.com

${ }^{1}$ School of Mathematics and

Statistics, Chongqing Three Gorges University, Chongqing, 404000, P.R. China

${ }^{2}$ Laboratory for Applied

Mathematics, Beijing

Computational Science Research Center, Beijing, 100084, P.R. China

Full list of author information is

available at the end of the article

\begin{abstract}
In this paper, we investigate new $L^{\infty}\left(L^{2}\right)$ and $L^{2}\left(L^{2}\right)$-posteriori error estimates of mixed finite element solutions for quadratic optimal control problems governed by semilinear parabolic equations. The state and the co-state are discretized by the order one Raviart-Thomas mixed finite element spaces and the control is approximated by piecewise constant functions. We derive a posteriori error estimates in $L^{\infty}\left(J ; L^{2}(\Omega)\right)$-norm and $L^{2}\left(J ; L^{2}(\Omega)\right)$-norm for both the state and the control approximation. Such estimates, which are apparently not available in the literature, are an important step towards developing reliable adaptive mixed finite element approximation schemes for the optimal control problem.
\end{abstract}

MSC: 49J20;65N30

Keywords: a posteriori error estimates; quadratic optimal control problems; semilinear parabolic equations; mixed finite element methods; integral constraint

\section{Introduction}

In this paper we consider quadratic optimal control problems governed by the semilinear parabolic equations

$$
\begin{aligned}
& \min _{u \in K \subset U}\left\{\frac{1}{2} \int_{0}^{T}\left(\left\|\mathbf{p}-\mathbf{p}_{d}\right\|^{2}+\left\|y-y_{d}\right\|^{2}+\|u\|^{2}\right) d t\right\}, \\
& y_{t}(x, t)+\operatorname{div} \mathbf{p}(x, t)+\phi(y(x, t))=f(x, t)+u(x, t), \quad x \in \Omega, t \in J, \\
& \mathbf{p}(x, t)=-A(x) \nabla y(x, t), \quad x \in \Omega, t \in J, \\
& y(x, t)=0, \quad x \in \partial \Omega, t \in J, \quad y(x, 0)=y_{0}(x), \quad x \in \Omega,
\end{aligned}
$$

where the bounded open set $\Omega \subset \mathbf{R}^{2}$ is a convex polygon with the boundary $\partial \Omega . J=[0, T]$. Let $K$ be a closed convex set in the control space $U=L^{2}\left(J ; L^{2}(\Omega)\right), \mathbf{p}, \mathbf{p}_{d} \in\left(L^{2}\left(J ; H^{1}(\Omega)\right)\right)^{2}$, $y, y_{d} \in L^{2}\left(J ; H^{1}(\Omega)\right), f, u \in L^{2}\left(J ; L^{2}(\Omega)\right), y_{0}(x) \in H_{0}^{1}(\Omega)$. For any $R>0$, the function $\phi(\cdot) \in$ $W^{1, \infty}(-R, R), \phi^{\prime}(y) \in L^{2}(\Omega)$ for any $y \in H^{1}(\Omega)$, and $\phi^{\prime}(y) \geq 0$. Assume that the coefficient matrix $A(x)=\left(a_{i j}(x)\right)_{2 \times 2} \in C^{\infty}\left(\bar{\Omega} ; \mathbf{R}^{2 \times 2}\right)$ is a symmetric $2 \times 2$-matrix and there are constants $c_{1}, c_{2}>0$ satisfying for any vector $\mathbf{X} \in \mathbf{R}^{2}, c_{1}\|\mathbf{X}\|_{\mathbf{R}^{2}}^{2} \leq \mathbf{X}^{t} A \mathbf{X} \leq c_{2}\|\mathbf{X}\|_{\mathbf{R}^{2}}^{2}$. We assume

@2013 Lu et al: licensee Springer. This is an Open Access article distributed under the terms of the Creative Commons Attribution License (http://creativecommons.org/licenses/by/2.0), which permits unrestricted use, distribution, and reproduction in any medium, provided the original work is properly cited. 
that the constraint on the control is an obstacle such that

$$
K=\left\{u \in L^{2}\left(J ; L^{2}(\Omega)\right): \int_{\Omega} u(x, t) d x \geq 0 \text {, a.e. in } \Omega \times J\right\} .
$$

Optimal control problems have been successfully utilized in scientific and engineering numerical simulation. Thus they must be solved by using some efficient numerical methods. Among these numerical methods, the finite element method was a good choice for solving partial differential equations. There have been extensive studies in convergence for finite element approximation of optimal control problems. A systematic introduction of the finite element method for optimal control problems can be found in [1-7].

Recently, an adaptive finite element method has been investigated extensively. It has become one of the most popular methods in the scientific computation and numerical modeling. Adaptive finite element approximation ensures a higher density of nodes in a certain area of the given domain, where the solution is more difficult to approximate, indicated by a posteriori error estimators. Hence it is an important approach to boost the accuracy and efficiency of finite element discretizations. There are lots of works concentrating on the adaptivity of many optimal control problems, for example, [8-12]. Note that all the above works aimed at the standard finite element method.

In many control problems, the objective functional contains the gradient of state variables. Thus, the accuracy of the gradient is important in numerical discretization of the coupled state equations. Mixed finite element methods are appropriate for the state equations in such cases since both the scalar variable and its flux variable can be approximated to the same accuracy by using such methods. When the objective functional contains the gradient of the state variable, mixed finite element methods should be used for discretization of the state equation with which both the scalar variable and its flux variable can be approximated in the same accuracy.

Recently, in $[13,14]$ we did some primary work on a priori error estimates for nonlinear parabolic optimal control problems by mixed finite element methods. In [15], we considered a posteriori error estimates of triangular mixed finite element methods for semilinear elliptic optimal control problems. The state and the co-state were discretized by the Raviart-Thomas mixed finite element spaces and the control was approximated by piecewise constant functions. In [16], we derived a posteriori error estimates for linear parabolic optimal control problems by the lowest order Raviart-Thomas mixed finite element methods.

This paper is motivated by the idea of the article [17]. We shall use the order one RaviartThomas mixed finite element to discretize the state and the co-state. Due to the limited regularity of the optimal control $u$ in general, we therefore only consider a piecewise constant space. Then we derive $a$ posteriori error estimates for the mixed finite element approximation of the optimal control problem. The estimators for the control, the state and the co-state variables are derived in the sense of $L^{\infty}\left(J ; L^{2}(\Omega)\right)$-norm or $L^{2}\left(J ; L^{2}(\Omega)\right)$-norm, which are different from the ones in [16].

In this paper, we adopt the standard notation $W^{m, p}(\Omega)$ for Sobolev spaces on $\Omega$ with a norm $\|\cdot\|_{m, p}$ given by $\|v\|_{m, p}^{p}=\sum_{|\alpha| \leq m}\left\|D^{\alpha} v\right\|_{L^{p}(\Omega)}^{p}$, a semi-norm $|\cdot|_{m, p}$ given by $|v|_{m, p}^{p}=$ $\sum_{|\alpha|=m}\left\|D^{\alpha} v\right\|_{L^{p}(\Omega)}^{p}$. We set $W_{0}^{m, p}(\Omega)=\left\{v \in W^{m, p}(\Omega):\left.v\right|_{\partial \Omega}=0\right\}$. For $p=2$, we define $H^{m}(\Omega)=W^{m, 2}(\Omega), H_{0}^{m}(\Omega)=W_{0}^{m, 2}(\Omega)$, and $\|\cdot\|_{m}=\|\cdot\|_{m, 2},\|\cdot\|=\|\cdot\|_{0,2}$. We denote by $L^{s}\left(0, T ; W^{m, p}(\Omega)\right)$ the Banach space of all $L^{s}$ integrable functions from $J$ into $W^{m, p}(\Omega)$ with 
the norm $\|v\|_{L^{s}\left(J ; W^{m, p}(\Omega)\right)}=\left(\int_{0}^{T}\|v\|_{W^{m, p}(\Omega)}^{s} d t\right)^{\frac{1}{s}}$ for $s \in[1, \infty)$, and the standard modification for $s=\infty$. Similarly, one can define the spaces $H^{1}\left(J ; W^{m, p}(\Omega)\right)$ and $C^{k}\left(J ; W^{m, p}(\Omega)\right)$. The details can be found in [18].

The plan of this paper is as follows. In the next section, we shall construct the mixed finite element approximation and the backward Euler discretization for quadratic optimal control problems governed by semilinear parabolic equations (1.1)-(1.4). Then, we derive a posteriori error estimates for both the state and the control approximation in Section 3. Finally, we give a conclusion and some future work.

\section{Mixed methods of optimal control problems}

In this section we shall now discuss the mixed finite element approximation and the backward Euler discretization of quadratic semilinear parabolic optimal control problems (1.1)(1.4). To fix the idea, we shall take the state spaces $L^{2}(\mathbf{V})=L^{2}(J ; \mathbf{V})$ and $H^{1}(W)=H^{1}(J ; W)$, where $\mathbf{V}$ and $W$ are defined as follows:

$$
\mathbf{V}=H(\operatorname{div} ; \Omega)=\left\{\mathbf{v} \in\left(L^{2}(\Omega)\right)^{2}, \operatorname{div} \mathbf{v} \in L^{2}(\Omega)\right\}, \quad W=L^{2}(\Omega) .
$$

The Hilbert space $\mathbf{V}$ is equipped with the following norm:

$$
\|\mathbf{v}\|_{H(\mathrm{div} ; \Omega)}=\left(\|\mathbf{v}\|_{0, \Omega}^{2}+\|\operatorname{div} \mathbf{v}\|_{0, \Omega}^{2}\right)^{1 / 2} .
$$

Let $\alpha=A^{-1}$, we recast (1.1)-(1.4) as the following weak form: find $(\mathbf{p}, y, u) \in L^{2}(\mathbf{V}) \times$ $H^{1}(W) \times K$ such that

$$
\begin{aligned}
& \min _{u \in K \subset U}\left\{\frac{1}{2} \int_{0}^{T}\left(\left\|\mathbf{p}-\mathbf{p}_{d}\right\|^{2}+\left\|y-y_{d}\right\|^{2}+\|u\|^{2}\right) d t\right\}, \\
& (\alpha \mathbf{p}, \mathbf{v})-(y, \operatorname{div} \mathbf{v})=0, \quad \forall \mathbf{v} \in \mathbf{V}, \\
& \left(y_{t}, w\right)+(\operatorname{div} \mathbf{p}, w)+(\phi(y), w)=(f+u, w), \quad \forall w \in W, \\
& y(x, 0)=y_{0}(x), \quad \forall x \in \Omega .
\end{aligned}
$$

It follows from [17] that optimal control problem (2.1)-(2.4) has a solution (p, $y, u)$, and that if a triplet $(\mathbf{p}, y, u)$ is the solution of (2.1)-(2.4), then there is a co-state $(\mathbf{q}, z) \in L^{2}(\mathbf{V}) \times$ $H^{1}(W)$ such that $(\mathbf{p}, y, \mathbf{q}, z, u)$ satisfies the following optimality conditions:

$$
\begin{aligned}
& (\alpha \mathbf{p}, \mathbf{v})-(y, \operatorname{div} \mathbf{v})=0, \quad \forall \mathbf{v} \in \mathbf{V}, \\
& \left(y_{t}, w\right)+(\operatorname{div} \mathbf{p}, w)+(\phi(y), w)=(f+u, w), \quad \forall w \in W, \\
& y(x, 0)=y_{0}(x), \quad \forall x \in \Omega, \\
& (\alpha \mathbf{q}, \mathbf{v})-(z, \operatorname{div} \mathbf{v})=-\left(\mathbf{p}-\mathbf{p}_{d}, \mathbf{v}\right), \quad \forall \mathbf{v} \in \mathbf{V}, \\
& -\left(z_{t}, w\right)+(\operatorname{div} \mathbf{q}, w)+\left(\phi^{\prime}(y) z, w\right)=\left(y-y_{d}, w\right), \quad \forall w \in W, \\
& z(x, T)=0, \quad \forall x \in \Omega, \\
& \int_{0}^{T}(u+z, \tilde{u}-u) d t \geq 0, \quad \forall \tilde{u} \in K,
\end{aligned}
$$

where $(\cdot, \cdot)$ is the inner product of $L^{2}(\Omega)$. 
In [19], the expression of the control variable was given. Here, we adopt the same method to derive the following operator:

$$
u=\max \{0, \bar{z}\}-z
$$

where $\bar{z}=\int_{\Omega} z / \int_{\Omega} 1 d x$ denotes the integral average on $\Omega$ of the function $z$.

Let $\mathcal{T}_{h}$ be regular triangulations of $\Omega . h_{\tau}$ is the diameter of $\tau$ and $h=\max h_{\tau}$. Let $\mathbf{V}_{h} \times W_{h} \subset \mathbf{V} \times W$ denote the order one Raviart-Thomas space associated with the triangulations $\mathcal{T}_{h}$ of $\Omega$. $P_{k}$ denotes the space of polynomials of total degree at most $k$. Let $\mathbf{V}(\tau)=\left\{\mathbf{v} \in P_{1}^{2}(\tau)+x \cdot P_{1}(\tau)\right\}, W(\tau)=P_{1}(\tau)$. We define

$$
\begin{aligned}
& \mathbf{V}_{h}:=\left\{\mathbf{v}_{h} \in \mathbf{V}: \forall \tau \in \mathcal{T}_{h},\left.\mathbf{v}_{h}\right|_{\tau} \in \mathbf{V}(\tau)\right\}, \\
& W_{h}:=\left\{w_{h} \in W: \forall \tau \in \mathcal{T}_{h},\left.w_{h}\right|_{\tau} \in W(\tau)\right\}, \\
& K_{h}:=\left\{\tilde{u}_{h} \in K: \forall \tau \in \mathcal{T}_{h},\left.\tilde{u}_{h}\right|_{\tau} \in P_{0}(\tau)\right\} .
\end{aligned}
$$

Let $L^{2}\left(\mathbf{V}_{h}\right)=L^{2}\left(J ; \mathbf{V}_{h}\right)$ and $H^{1}\left(W_{h}\right)=H^{1}\left(J ; W_{h}\right)$. The mixed finite element discretization of (2.1)-(2.4) is as follows: compute $\left(\mathbf{p}_{h}, y_{h}, u_{h}\right) \in L^{2}\left(\mathbf{V}_{h}\right) \times H^{1}\left(W_{h}\right) \times K_{h}$ such that

$$
\begin{aligned}
& \min _{u_{h} \in K_{h}}\left\{\frac{1}{2} \int_{0}^{T}\left(\left\|\mathbf{p}_{h}-\mathbf{p}_{d}\right\|^{2}+\left\|y_{h}-y_{d}\right\|^{2}+\left\|u_{h}\right\|^{2}\right) d t\right\}, \\
& \left(\alpha \mathbf{p}_{h}, \mathbf{v}_{h}\right)-\left(y_{h}, \operatorname{div} \mathbf{v}_{h}\right)=0, \quad \forall \mathbf{v}_{h} \in \mathbf{V}_{h}, \\
& \left(y_{h t}, w_{h}\right)+\left(\operatorname{div} \mathbf{p}_{h}, w_{h}\right)+\left(\phi\left(y_{h}\right), w_{h}\right)=\left(f+u_{h}, w_{h}\right), \quad \forall w_{h} \in W_{h}, \\
& y_{h}(x, 0)=y_{0}^{h}(x), \quad \forall x \in \Omega,
\end{aligned}
$$

where $y_{0}^{h}(x) \in W_{h}$ is an approximation of $y_{0}$. Optimal control problem (2.13)-(2.16) again has a solution $\left(\mathbf{p}_{h}, y_{h}, u_{h}\right)$, and that if a triplet $\left(\mathbf{p}_{h}, y_{h}, u_{h}\right)$ is the solution of (2.13)-(2.16), then there is a co-state $\left(\mathbf{q}_{h}, z_{h}\right) \in L^{2}\left(\mathbf{V}_{h}\right) \times H^{1}\left(W_{h}\right)$ such that $\left(\mathbf{p}_{h}, y_{h}, \mathbf{q}_{h}, z_{h}, u_{h}\right)$ satisfies the following optimality conditions:

$$
\begin{aligned}
& \left(\alpha \mathbf{p}_{h}, \mathbf{v}_{h}\right)-\left(y_{h}, \operatorname{div} \mathbf{v}_{h}\right)=0, \quad \forall \mathbf{v}_{h} \in \mathbf{V}_{h}, \\
& \left(y_{h t}, w_{h}\right)+\left(\operatorname{div} \mathbf{p}_{h}, w_{h}\right)+\left(\phi\left(y_{h}\right), w_{h}\right)=\left(f+u_{h}, w_{h}\right), \quad \forall w_{h} \in W_{h}, \\
& y_{h}(x, 0)=y_{0}^{h}(x), \quad \forall x \in \Omega, \\
& \left(\alpha \mathbf{q}_{h}, \mathbf{v}_{h}\right)-\left(z_{h}, \operatorname{div} \mathbf{v}_{h}\right)=-\left(\mathbf{p}_{h}-\mathbf{p}_{d}, \mathbf{v}_{h}\right), \quad \forall \mathbf{v}_{h} \in \mathbf{V}_{h}, \\
& -\left(z_{h t}, w_{h}\right)+\left(\operatorname{div} \mathbf{q}_{h}, w_{h}\right)+\left(\phi^{\prime}\left(y_{h}\right) z_{h}, w_{h}\right)=\left(y_{h}-y_{d}, w_{h}\right), \quad \forall w_{h} \in W_{h}, \\
& z_{h}(x, T)=0, \quad \forall x \in \Omega, \\
& \int_{0}^{T}\left(u_{h}+z_{h}, \tilde{u}_{h}-u_{h}\right) d t \geq 0, \quad \forall \tilde{u}_{h} \in K_{h} .
\end{aligned}
$$

Next, we define the standard $L^{2}(\Omega)$-orthogonal projection $\mathcal{Q}_{h}: K \rightarrow K_{h}$, which satisfies: for any $\tilde{u} \in K$,

$$
\left(\tilde{u}-\mathcal{Q}_{h} \tilde{u}, \tilde{u}_{h}\right)=0, \quad \forall \tilde{u}_{h} \in K_{h},
$$




$$
\left\|\tilde{u}-\mathcal{Q}_{h} \tilde{u}\right\|_{-s, r} \leq C|\tilde{u}|_{1, r} h^{1+s}, \quad s=0,1 \text { for } \tilde{u} \in W^{1, s}(\Omega)
$$

Similar to (2.12), for variational inequality (2.23), we have the following conclusion [19]. Assume that $z_{h}$ is known in variational inequality (2.23). The solution of the variational inequality is

$$
u_{h}=\mathcal{Q}_{h}\left(\max \left\{0, \bar{z}_{h}\right\}-z_{h}\right), \quad \bar{z}_{h}=\frac{\int_{\Omega} z_{h} d x}{\int_{\Omega} 1 d x} .
$$

Now we consider the fully discrete approximation for the above semidiscrete problem. Let $\Delta t>0, N=\frac{T}{\Delta t} \in \mathbb{Z}$, and $t_{i}=i \Delta t, i \in \mathbb{Z}$. Also, let

$$
\psi^{i}=\psi^{i}(x)=\psi\left(x, t_{i}\right), \quad d_{t} \psi^{i}=\frac{\psi^{i}-\psi^{i-1}}{\Delta t} .
$$

The following fully discrete approximation scheme is to find $\left(\mathbf{p}_{h}^{i}, y_{h}^{i}, u_{h}^{i}\right) \in \mathbf{V}_{h} \times W_{h} \times K_{h}$, $i=1,2, \ldots, N$, such that

$$
\begin{aligned}
& \min _{u_{h}^{i} \in K_{h}}\left\{\frac{1}{2} \sum_{i=1}^{N} \Delta t\left(\left\|\mathbf{p}_{h}^{i}-\mathbf{p}_{d}^{i}\right\|^{2}+\left\|y_{h}^{i}-y_{d}^{i}\right\|^{2}+\left\|u_{h}^{i}\right\|^{2}\right)\right\}, \\
& \left(\alpha \mathbf{p}_{h}^{i}, \mathbf{v}_{h}\right)-\left(y_{h}^{i}, \operatorname{div} \mathbf{v}_{h}\right)=0, \quad \forall \mathbf{v}_{h} \in \mathbf{V}_{h}, \\
& \left(d_{t} y_{h}^{i}, w_{h}\right)+\left(\operatorname{div} \mathbf{p}_{h}^{i}, w_{h}\right)+\left(\phi\left(y_{h}^{i}\right), w_{h}\right)=\left(f^{i}+u_{h}^{i}, w_{h}\right), \quad \forall w_{h} \in W_{h}, \\
& y_{h}^{0}(x)=y_{0}^{h}(x), \quad \forall x \in \Omega .
\end{aligned}
$$

It follows that optimal control problem (2.27)-(2.30) has a solution $\left(\mathbf{p}_{h}^{i}, y_{h}^{i}, u_{h}^{i}\right), i=$ $1,2, \ldots, N$, and that if a triplet $\left(\mathbf{p}_{h}^{i}, y_{h}^{i}, u_{h}^{i}\right) \in \mathbf{V}_{h} \times W_{h} \times K_{h}, i=1,2, \ldots, N$, is the solution of (2.27)-(2.30), then there is a co-state $\left(\mathbf{q}_{h}^{i-1}, z_{h}^{i-1}\right) \in \mathbf{V}_{h} \times W_{h}$ such that $\left(\mathbf{p}_{h}^{i}, y_{h}^{i}, \mathbf{q}_{h}^{i-1}, z_{h}^{i-1}, u_{h}^{i}\right) \in$ $\left(\mathbf{V}_{h} \times W_{h}\right)^{2} \times K_{h}$ satisfies the following optimality conditions:

$$
\begin{aligned}
& \left(\alpha \mathbf{p}_{h}^{i}, \mathbf{v}_{h}\right)-\left(y_{h}^{i}, \operatorname{div} \mathbf{v}_{h}\right)=0, \quad \forall \mathbf{v}_{h} \in \mathbf{V}_{h}, \\
& \left(d_{t} y_{h}^{i}, w_{h}\right)+\left(\operatorname{div} \mathbf{p}_{h}^{i}, w_{h}\right)+\left(\phi\left(y_{h}^{i}\right), w_{h}\right)=\left(f^{i}+u_{h}^{i}, w_{h}\right), \quad \forall w_{h} \in W_{h}, \\
& y_{h}^{0}(x)=y_{0}^{h}(x), \quad \forall x \in \Omega, \\
& \left(\alpha \mathbf{q}_{h}^{i-1}, \mathbf{v}_{h}\right)-\left(z_{h}^{i-1}, \operatorname{div} \mathbf{v}_{h}\right)=-\left(\mathbf{p}_{h}^{i-1}-\mathbf{p}_{d}^{i-1}, \mathbf{v}_{h}\right), \quad \forall \mathbf{v}_{h} \in \mathbf{V}_{h}, \\
& -\left(d_{t} z_{h}^{i}, w_{h}\right)+\left(\operatorname{div} \mathbf{q}_{h}^{i-1}, w_{h}\right)+\left(\phi^{\prime}\left(y_{h}^{i}\right) z_{h}^{i-1}, w_{h}\right)=\left(y_{h}^{i-1}-y_{d}^{i-1}, w_{h}\right), \quad \forall w_{h} \in W_{h}, \\
& z_{h}^{N}(x)=0, \quad \forall x \in \Omega, \\
& \left(u_{h}^{i}+z_{h}^{i-1}, \tilde{u}_{h}-u_{h}^{i}\right) \geq 0, \quad \forall \tilde{u}_{h} \in K_{h} .
\end{aligned}
$$

For $i=1,2, \ldots, N$, let

$$
\begin{aligned}
& \left.Y_{h}\right|_{\left(t_{i-1}, t_{i}\right]}=\left(\left(t_{i}-t\right) y_{h}^{i-1}+\left(t-t_{i-1}\right) y_{h}^{i}\right) / \Delta t, \\
& \left.Z_{h}\right|_{\left(t_{i-1}, t_{i}\right]}=\left(\left(t_{i}-t\right) z_{h}^{i-1}+\left(t-t_{i-1}\right) z_{h}^{i}\right) / \Delta t, \\
& \left.P_{h}\right|_{\left(t_{i-1}, t_{i}\right]}=\left(\left(t_{i}-t\right) \mathbf{p}_{h}^{i-1}+\left(t-t_{i-1}\right) \mathbf{p}_{h}^{i}\right) / \Delta t,
\end{aligned}
$$




$$
\begin{aligned}
\left.Q_{h}\right|_{\left(t_{i-1}, t_{i}\right]} & =\left(\left(t_{i}-t\right) \mathbf{q}_{h}^{i-1}+\left(t-t_{i-1}\right) \mathbf{q}_{h}^{i}\right) / \Delta t, \\
\left.U_{h}\right|_{\left(t_{i-1}, t_{i}\right]} & =u_{h}^{i} .
\end{aligned}
$$

For any function $w \in C\left(J ; L^{2}(\Omega)\right)$, let

$$
\left.\hat{w}(x, t)\right|_{t \in\left(t_{i-1}, t_{i}\right]}=w\left(x, t_{i}\right),\left.\quad \tilde{w}(x, t)\right|_{t \in\left(t_{i-1}, t_{i}\right]}=w\left(x, t_{i-1}\right) .
$$

Then optimality conditions (2.31)-(2.37) satisfy

$$
\begin{aligned}
& \left(\alpha \hat{P}_{h}, \mathbf{v}_{h}\right)-\left(\hat{Y}_{h}, \operatorname{div} \mathbf{v}_{h}\right)=0, \quad \forall \mathbf{v}_{h} \in \mathbf{V}_{h}, \\
& \left(Y_{h t}, w_{h}\right)+\left(\operatorname{div} \hat{P}_{h}, w_{h}\right)+\left(\phi\left(\hat{Y}_{h}\right), w_{h}\right)=\left(\hat{f}+U_{h}, w_{h}\right), \quad \forall w_{h} \in W_{h}, \\
& Y_{h}(x, 0)=y_{0}^{h}(x), \quad \forall x \in \Omega, \\
& \left(\alpha \widetilde{Q}_{h}, \mathbf{v}_{h}\right)-\left(\widetilde{Z}_{h}, \operatorname{div} \mathbf{v}_{h}\right)=-\left(\widetilde{P}_{h}-\tilde{\mathbf{p}}_{d}, \mathbf{v}_{h}\right), \quad \forall \mathbf{v}_{h} \in \mathbf{V}_{h}, \\
& -\left(Z_{h t}, w_{h}\right)+\left(\operatorname{div} \widetilde{Q}_{h}, w_{h}\right)+\left(\phi^{\prime}\left(\hat{Y}_{h}\right) \widetilde{Z}_{h}, w_{h}\right)=\left(\widetilde{Y}_{h}-\tilde{y}_{d}, w_{h}\right), \quad \forall w_{h} \in W_{h}, \\
& Z_{h}(x, T)=0, \quad \forall x \in \Omega, \\
& \left(U_{h}+\widetilde{Z}_{h}, \tilde{u}_{h}-U_{h}\right) \geq 0, \quad \forall \tilde{u}_{h} \in K_{h} .
\end{aligned}
$$

Similar to (2.26), the solution of variational inequality (2.44) is

$$
U_{h}=\mathcal{Q}_{h}\left(\max \left\{0, \widetilde{Z}_{h}\right\}-\widetilde{Z}_{h}\right), \quad \widetilde{Z}_{h}=\frac{\int_{\Omega} \widetilde{Z}_{h} d x}{\int_{\Omega} 1 d x} .
$$

In the rest of the paper, we shall use some intermediate variables. For any control function $U_{h} \in K_{h}$, we first define the state solution $\left(\mathbf{p}\left(U_{h}\right), y\left(U_{h}\right), \mathbf{q}\left(U_{h}\right), z\left(U_{h}\right)\right)$ satisfying

$$
\begin{aligned}
& \left(\alpha \mathbf{p}\left(U_{h}\right), \mathbf{v}\right)-\left(y\left(U_{h}\right), \operatorname{div} \mathbf{v}\right)=0, \quad \forall \mathbf{v} \in \mathbf{V}, \\
& \left(y_{t}\left(U_{h}\right), w\right)+\left(\operatorname{div} \mathbf{p}\left(U_{h}\right), w\right)+\left(\phi\left(y\left(U_{h}\right)\right), w\right)=\left(f+U_{h}, w\right), \quad \forall w \in W, \\
& y\left(U_{h}\right)(x, 0)=y_{0}(x), \quad \forall x \in \Omega, \\
& \left(\alpha \mathbf{q}\left(U_{h}\right), \mathbf{v}\right)-\left(z\left(U_{h}\right), \operatorname{div} \mathbf{v}\right)=-\left(\mathbf{p}\left(U_{h}\right)-\mathbf{p}_{d}, \mathbf{v}\right), \quad \forall \mathbf{v} \in \mathbf{V}, \\
& -\left(z_{t}\left(U_{h}\right), w\right)+\left(\operatorname{div} \mathbf{q}\left(U_{h}\right), w\right)+\left(\phi^{\prime}\left(y\left(U_{h}\right)\right) z\left(U_{h}\right), w\right) \\
& \quad=\left(y\left(U_{h}\right)-y_{d}, w\right), \quad \forall w \in W \\
& z\left(U_{h}\right)(x, T)=0, \quad \forall x \in \Omega .
\end{aligned}
$$

For $\varphi \in W_{h}$, we shall write

$$
\phi(\varphi)-\phi(\rho)=-\tilde{\phi}^{\prime}(\varphi)(\rho-\varphi)=-\phi^{\prime}(\rho)(\rho-\varphi)+\tilde{\phi}^{\prime \prime}(\varphi)(\rho-\varphi)^{2},
$$

where

$$
\tilde{\phi}^{\prime}(\varphi)=\int_{0}^{1} \phi^{\prime}(\varphi+s(\rho-\varphi)) d s, \quad \tilde{\phi}^{\prime \prime}(\varphi)=\int_{0}^{1}(1-s) \phi^{\prime \prime}(\rho+s(\varphi-\rho)) d s
$$

are bounded functions in $\bar{\Omega}$. 
Let $R_{h}: W \rightarrow W_{h}$ be the orthogonal $L^{2}(\Omega)$-projection into $W_{h}[20]$ which satisfies:

$$
\begin{aligned}
& \left(R_{h} w-w, \chi\right)=0, \quad w \in W, \chi \in W_{h}, \\
& \left\|R_{h} w-w\right\|_{0, q} \leq C\|w\|_{t, q} h^{t}, \quad 0 \leq t \leq k+1, \text { if } w \in W \cap W^{t, q}(\Omega), \\
& \left\|R_{h} w-w\right\|_{-r} \leq C\|w\|_{t} h^{r+t}, \quad 0 \leq r, t \leq k+1, \text { if } w \in H^{t}(\Omega) .
\end{aligned}
$$

Let $\Pi_{h}: \mathbf{V} \rightarrow \mathbf{V}_{h}$ be the Raviart-Thomas projection operator [21] which satisfies: for any $\mathbf{v} \in \mathbf{V}$,

$$
\begin{array}{ll}
\int_{E} w_{h}\left(\mathbf{v}-\Pi_{h} \mathbf{v}\right) \cdot v_{E} d s=0, & w_{h} \in W_{h}, E \in \mathcal{E}_{h}, \\
\int_{T}\left(\mathbf{v}-\Pi_{h} \mathbf{v}\right) \cdot \mathbf{v}_{h} d x d y=0, & \mathbf{v}_{h} \in \mathbf{V}_{h}, T \in \mathcal{T}_{h},
\end{array}
$$

where $\mathcal{E}_{h}$ denote the set of element sides in $\mathcal{T}_{h}$. We have the commuting diagram property

$$
\operatorname{div} \circ \Pi_{h}=R_{h} \circ \operatorname{div}: \mathbf{V} \rightarrow W_{h} \text { and } \operatorname{div}\left(I-\Pi_{h}\right) \mathbf{V} \perp W_{h},
$$

where and after, $I$ denotes an identity matrix.

Further, the interpolation operator $\Pi_{h}$ satisfies a local error estimate

$$
\left\|\mathbf{v}-\Pi_{h} \mathbf{v}\right\|_{0, \Omega} \leq C h|\mathbf{v}|_{1, \mathcal{T}_{h}}, \quad \mathbf{v} \in \mathbf{V} \cap H^{1}\left(\mathcal{T}_{h}\right)
$$

The following lemmas are important in deriving a posteriori error estimates of residual type.

Lemma 2.1 Let $\hat{\pi}_{h}$ be the average interpolation operator defined in [22]. For $m=0$ or 1 , $1 \leq q \leq \infty$ and $\forall v \in W^{1, q}\left(\Omega^{h}\right)$,

$$
\left|v-\hat{\pi}_{h} v\right|_{W^{m, q}(\tau)} \leq \sum_{\bar{\tau}^{\prime} \cap \bar{\tau} \neq \emptyset} C h_{\tau}^{1-m}|v|_{W^{1, q}\left(\tau^{\prime}\right)} .
$$

Lemma 2.2 Let $\pi_{h}$ be the standard Lagrange interpolation operator [23]. Then, for $m=0$ or $1,1<q \leq \infty$ and $\forall v \in W^{2, q}\left(\Omega^{h}\right)$,

$$
\left|\nu-\pi_{h} \nu\right|_{W^{m, q}(\tau)} \leq C h_{\tau}^{2-m}|\nu|_{W^{2, q}(\tau)} .
$$

\section{A posteriori error estimates}

In this section we study new $L^{\infty}\left(L^{2}\right)$ and $L^{2}\left(L^{2}\right)$-posteriori error estimates for the mixed finite element approximation to the semilinear parabolic optimal control problems. Let

$$
\begin{aligned}
& S(u)=\frac{1}{2}\left(\left\|\mathbf{p}-\mathbf{p}_{d}\right\|^{2}+\left\|y-y_{d}\right\|^{2}+\|u\|^{2}\right), \\
& S_{h}\left(U_{h}\right)=\frac{1}{2}\left(\left\|P_{h}-\mathbf{p}_{d}\right\|^{2}+\left\|Y_{h}-y_{d}\right\|^{2}+\left\|U_{h}\right\|^{2}\right) .
\end{aligned}
$$

It can be shown that (see [13] for some detail discussions)

$$
\left(S^{\prime}(u), v\right)=(u+z, v)
$$




$$
\begin{aligned}
& \left(S^{\prime}\left(U_{h}\right), v\right)=\left(U_{h}+z\left(U_{h}\right), v\right), \\
& \left(S_{h}^{\prime}\left(U_{h}\right), v\right)=\left(U_{h}+\widetilde{Z}_{h}, v\right) .
\end{aligned}
$$

It is clear that $S$ and $S_{h}$ are well defined and continuous on $K$ and $K_{h}$. Also, the functional $S_{h}$ can be naturally extended on $K$. Then (2.1) and (2.27) can be represented as

$$
\min _{u \in K}\left\{\int_{0}^{T} S(u) d t\right\}
$$

and

$$
\min _{U_{h} \in K_{h}}\left\{\int_{0}^{T} S_{h}\left(U_{h}\right) d t\right\}
$$

In many applications, $S(\cdot)$ is uniform convex near the solution $u$. The convexity of $S(\cdot)$ is closely related to the second-order sufficient conditions of the optimal control problems, which are assumed in many studies on numerical methods of the problem. For instance, in many applications, there is $c>0$, independent of $h$, such that

$$
\int_{0}^{T}\left(S^{\prime}(u)-S^{\prime}\left(U_{h}\right), u-U_{h}\right)_{U} \geq c\left\|u-U_{h}\right\|_{L^{2}\left(J ; L^{2}(\Omega)\right)}^{2}
$$

Firstly, let us derive a posteriori error estimates for the control $u$.

Theorem 3.1 Let $u$ and $U_{h}$ be the solutions of (3.6) and (3.7), respectively. Assume that $\left.\left(S_{h}^{\prime}\left(U_{h}\right)\right)\right|_{\tau} \in H^{s}(\tau), \forall \tau \in \mathcal{T}_{h}(s=0,1)$, and there is $v_{h} \in K_{h}$ such that

$$
\left|\left(S_{h}^{\prime}\left(U_{h}\right), v_{h}-u\right)\right| \leq C \sum_{\tau \in \mathcal{T}_{h}} h_{\tau}\left\|S_{h}^{\prime}\left(U_{h}\right)\right\|_{H^{s}(\tau)}\left\|u-U_{h}\right\|_{L^{2}(\tau)}^{s} .
$$

Then we have

$$
\left\|u-U_{h}\right\|_{L^{2}\left(J ; L^{2}(\Omega)\right)}^{2} \leq C \eta_{1}^{2}+C\left\|z\left(U_{h}\right)-\widetilde{Z}_{h}\right\|_{L^{2}\left(j ; L^{2}(\Omega)\right)}^{2},
$$

where

$$
\eta_{1}^{2}=\int_{0}^{T} \sum_{\tau \in \mathcal{T}_{h}} h_{\tau}^{1+s}\left\|U_{h}+\widetilde{Z}_{h}\right\|_{H^{1}(\tau)}^{1+s} d t
$$

Proof It follows from (3.6) and (3.7) that

$$
\begin{aligned}
& \int_{0}^{T}\left(S^{\prime}(u), u-v\right) \leq 0, \quad \forall v \in K, \\
& \int_{0}^{T}\left(S_{h}^{\prime}\left(U_{h}\right), U_{h}-v_{h}\right) \leq 0, \quad \forall v_{h} \in K_{h} \subset K .
\end{aligned}
$$


Then it follows from assumptions (3.8), (3.9), and the Schwarz inequality that

$$
\begin{aligned}
c \| u & -U_{h} \|_{L^{2}\left(j ; L^{2}(\Omega)\right)}^{2} \\
\leq & \int_{0}^{T}\left(S^{\prime}(u)-S^{\prime}\left(U_{h}\right), u-U_{h}\right) \\
\leq & \int_{0}^{T}\left\{\left(S_{h}^{\prime}\left(U_{h}\right), v_{h}-u\right)+\left(S_{h}^{\prime}\left(U_{h}\right)-S^{\prime}\left(U_{h}\right), u-U_{h}\right)\right\} \\
\leq & C \int_{0}^{T}\left\{\sum_{\tau \in \mathcal{T}_{h}} h_{\tau}^{1+s}\left\|S_{h}^{\prime}\left(U_{h}\right)\right\|_{H^{s}(\tau)}^{1+s}\right. \\
& \left.+\left\|S_{h}^{\prime}\left(U_{h}\right)-S^{\prime}\left(U_{h}\right)\right\|_{L^{2}(\Omega)}^{2}\right\}+\frac{c}{2}\left\|u-U_{h}\right\|_{L^{2}\left(j ; L^{2}(\Omega)\right)}^{2} .
\end{aligned}
$$

It is not difficult to show

$$
S_{h}^{\prime}\left(U_{h}\right)=U_{h}+\widetilde{Z}_{h}, \quad S^{\prime}\left(U_{h}\right)=U_{h}+z\left(U_{h}\right),
$$

where $z\left(U_{h}\right)$ is defined in (2.46)-(2.51). Thanks to (3.14), it is easy to derive

$$
\left\|S_{h}^{\prime}\left(U_{h}\right)-S^{\prime}\left(U_{h}\right)\right\|_{L^{2}(\Omega)}=\left\|\widetilde{Z}_{h}-z\left(U_{h}\right)\right\|_{L^{2}(\Omega)} .
$$

Then, by estimates (3.13) and (3.15), we can prove the requested result (3.10).

To estimate the error $\left\|\widetilde{Z}_{h}-z\left(U_{h}\right)\right\|_{L^{2}\left(j ; L^{2}(\Omega)\right)}^{2}$, we need the following well-known stability results for the following dual equations:

$$
\left\{\begin{array}{l}
-\kappa_{t}-\operatorname{div}(A \nabla \kappa)+\lambda \kappa=0, \quad x \in \Omega, t \in\left[0, t^{*}\right] \\
\left.\kappa\right|_{\partial \Omega}=0, \quad t \in\left[0, t^{*}\right] \\
\kappa\left(x, t^{*}\right)=\kappa_{0}(x), \quad x \in \Omega
\end{array}\right.
$$

and

$$
\left\{\begin{array}{l}
\varpi_{t}-\operatorname{div}\left(A^{*} \nabla \varpi\right)+\phi^{\prime}\left(y\left(U_{h}\right)\right) \varpi=0, \quad x \in \Omega, t \in\left[t^{*}, T\right] \\
\left.\varpi\right|_{\partial \Omega}=0, \quad t \in\left[t^{*}, T\right] \\
\varpi\left(x, t^{*}\right)=\varpi_{0}(x), \quad x \in \Omega,
\end{array}\right.
$$

where

$$
\lambda= \begin{cases}\frac{\phi\left(y\left(U_{h}\right)\right)-\phi\left(Y_{h}\right)}{y\left(U_{h}\right)-Y_{h}}, & y\left(U_{h}\right) \neq Y_{h} \\ \phi^{\prime}\left(Y_{h}\right), & y\left(U_{h}\right)=Y_{h} .\end{cases}
$$


Lemma 3.1 Let $\kappa$ and $\varpi$ be the solutions of (3.16) and (3.17), respectively $[24,25]$. Let $\Omega$ be a convex domain. Then

$$
\begin{aligned}
& \int_{\Omega}|\kappa(x, t)|^{2} d x \leq C\left\|\kappa_{0}\right\|_{L^{2}(\Omega)}^{2}, \quad \forall t \in\left[0, t^{*}\right], \\
& \int_{0}^{t^{*}} \int_{\Omega}|\nabla \kappa|^{2} d x d t \leq C\left\|\kappa_{0}\right\|_{L^{2}(\Omega)}^{2}, \\
& \int_{0}^{t^{*}} \int_{\Omega}\left|t-t^{*}\right|\left|D^{2} \kappa\right|^{2} d x d t \leq C\left\|\kappa_{0}\right\|_{L^{2}(\Omega)}^{2} \\
& \int_{0}^{t^{*}} \int_{\Omega}\left|t-t^{*}\right|\left|\kappa_{t}\right|^{2} d x d t \leq C\left\|\kappa_{0}\right\|_{L^{2}(\Omega)}^{2},
\end{aligned}
$$

and

$$
\begin{aligned}
& \int_{\Omega}|\varpi(x, t)|^{2} d x \leq C\left\|\varpi_{0}\right\|_{L^{2}(\Omega)}^{2}, \quad \forall t \in\left[t^{*}, T\right], \\
& \int_{t^{*}}^{T} \int_{\Omega}|\nabla \varpi|^{2} d x d t \leq C\left\|\varpi_{0}\right\|_{L^{2}(\Omega)}^{2} \\
& \int_{t^{*}}^{T} \int_{\Omega}\left|t-t^{*}\right|\left|D^{2} \varpi\right|^{2} d x d t \leq C\left\|\varpi_{0}\right\|_{L^{2}(\Omega)}^{2} \\
& \int_{t^{*}}^{T} \int_{\Omega}\left|t-t^{*}\right|\left|\varpi_{t}\right|^{2} d x d t \leq C\left\|\varpi_{0}\right\|_{L^{2}(\Omega)}^{2}
\end{aligned}
$$

where $\left|D^{2} v\right|=\max \left\{\left|\partial^{2} v / \partial x_{i} \partial x_{j}\right|, 1 \leq i, j \leq 2\right\}$.

Next, we estimate the errors $Y_{h}-y\left(U_{h}\right)$ and $P_{h}-\mathbf{p}\left(U_{h}\right)$.

Theorem 3.2 Let $\left(P_{h}, Y_{h}, Q_{h}, Z_{h}, U_{h}\right)$ and $\left(\mathbf{p}\left(U_{h}\right), y\left(U_{h}\right), \mathbf{q}\left(U_{h}\right), z\left(U_{h}\right), U_{h}\right)$ be the solutions of (2.38)-(2.44) and (2.46)-(2.51), respectively. Then we have

$$
\left\|Y_{h}-y\left(U_{h}\right)\right\|_{L^{\infty}\left(j L^{2}(\Omega)\right)}^{2}+\left\|P_{h}-\mathbf{p}\left(U_{h}\right)\right\|_{L^{2}\left(j ; L^{2}(\Omega)\right)} \leq C \sum_{i=2}^{7} \eta_{i}^{2},
$$

where

$$
\begin{aligned}
& \eta_{2}^{2}=\max _{i \in[1, N-1]}\left\{\int_{t_{i-1}}^{t_{i+1}} \sum_{\tau} h_{\tau}^{2} \int_{\tau}\left(Y_{h t}+\operatorname{div} \hat{P}_{h}+\phi\left(\hat{Y}_{h}\right)-\hat{f}-U_{h}\right)^{2} d x d t\right\} ; \\
& \eta_{3}^{2}=\max _{i \in[1, N-1]}\left\{\int_{t_{i-1}}^{t_{i+1}} \sum_{\tau} \min _{w_{h} \in W_{h}} \int_{\tau}\left(\alpha P_{h}-\nabla w_{h}\right)^{2} d x d t\right\} ; \\
& \eta_{4}^{2}=|\ln \Delta t| \max _{t \in[0, T]}\left\{\sum_{\tau} h_{\tau}^{4} \int_{\tau}\left(Y_{h t}+\operatorname{div} \hat{P}_{h}+\phi\left(\hat{Y}_{h}\right)-\hat{f}-U_{h}\right)^{2} d x\right\} ; \\
& \eta_{5}^{2}=|\ln \Delta t| \max _{t \in[0, T]}\left\{\sum_{\tau} h_{\tau}^{2} \cdot \min _{w_{h} \in W_{h}} \int_{\tau}\left(\alpha P_{h}-\nabla w_{h}\right)^{2} d x\right\} ; \\
& \eta_{6}^{2}=\|\hat{f}-f\|_{L^{1}\left(0, t^{*} ; L^{2}(\Omega)\right)}^{2}+\left\|y_{0}^{h}(x)-y_{0}(x)\right\|_{L^{2}(\Omega)}^{2} ; \\
& \eta_{7}^{2}=\left\|\hat{P}_{h}-P_{h}\right\|_{L^{2}\left(0, t^{*} ; L^{2}(\Omega)\right)}^{2}+\left\|\hat{Y}_{h}-Y_{h}\right\|_{L^{2}\left(0, t^{*} ; L^{2}(\Omega)\right)^{*}}^{2}
\end{aligned}
$$


Proof We define $\mathbf{p}_{h}^{N}$ as follows:

$$
\left(\alpha \mathbf{p}_{h}^{N}, \mathbf{v}_{h}\right)-\left(y_{h}^{N}, \operatorname{div} \mathbf{v}_{h}\right)=0, \quad \forall \mathbf{v}_{h} \in \mathbf{V}_{h}
$$

Then from (3.20) we deduce that

$$
\left(\alpha \mathbf{p}_{h}^{N-1}, \mathbf{v}_{h}\right)-\left(y_{h}^{N-1}, \operatorname{div} \mathbf{v}_{h}\right)=0, \quad \forall \mathbf{v}_{h} \in \mathbf{V}_{h}
$$

Combining (3.20)-(3.21) and the definitions of $Y_{h}$ and $P_{h}$, we can get the following equality:

$$
\left(\alpha P_{h}, \mathbf{v}_{h}\right)-\left(Y_{h}, \operatorname{div} \mathbf{v}_{h}\right)=0, \quad \forall \mathbf{v}_{h} \in \mathbf{V}_{h}
$$

Let $\kappa$ be the solution of (3.16) with $\kappa_{0}(x)=\left(Y_{h}-y\left(U_{h}\right)\right)\left(x, t^{*}\right)$, we infer that

$$
\begin{aligned}
\left\|Y_{h}-y\left(U_{h}\right)\right\|_{L^{2}(\Omega)}^{2} & \left(\left(Y_{h}-y\left(U_{h}\right)\right)\left(x, t^{*}\right), \kappa\left(x, t^{*}\right)\right) \\
= & \int_{0}^{t^{*}}\left(\left(\left(Y_{h}-y\left(U_{h}\right)\right)_{t}, \kappa\right)-\left(Y_{h}-y\left(U_{h}\right), \operatorname{div}(A \nabla \kappa)\right)\right) d t \\
& +\int_{0}^{t^{*}}\left(\phi\left(Y_{h}\right)-\phi\left(y\left(U_{h}\right)\right), \kappa\right) d t+\left(\left(Y_{h}-y\left(U_{h}\right)\right)(x, 0), \kappa(x, 0)\right) \\
= & \int_{0}^{t^{*}}\left(\left(\left(Y_{h}-y\left(U_{h}\right)\right)_{t}, \kappa\right)+\left(p\left(U_{h}\right), \nabla \kappa\right)\right) d t \\
& -\int_{0}^{t^{*}}\left(Y_{h}, \operatorname{div}\left(\Pi_{h}(A \nabla \kappa)\right)\right) d t-\int_{0}^{t^{*}}\left(\phi\left(y\left(U_{h}\right)\right), \kappa\right) d t \\
& +\int_{0}^{t^{*}}\left(\phi\left(Y_{h}\right), \kappa\right) d t+\left(\left(Y_{h}-y\left(U_{h}\right)\right)(x, 0), \kappa(x, 0)\right) .
\end{aligned}
$$

Furthermore, using (2.38)-(2.40), (2.46)-(2.48) and (2.56)-(2.58), we can obtain that

$$
\begin{aligned}
\left\|Y_{h}-y\left(U_{h}\right)\right\|_{L^{2}(\Omega)}^{2} & \int_{0}^{t^{*}}\left(\left(\left(Y_{h}-y\left(U_{h}\right)\right)_{t^{\prime}},\right)+\left(\operatorname{div}\left(\hat{P}_{h}-p\left(U_{h}\right)\right), \kappa\right)\right) d t \\
& +\int_{0}^{t^{*}}\left(\left(\alpha P_{h}, \Pi_{h}(A \nabla \kappa)\right)-\left(\operatorname{div} \hat{P}_{h}, \kappa\right)\right) d t-\int_{0}^{t^{*}}\left(\phi\left(y\left(U_{h}\right)\right), \kappa\right) d t \\
& +\int_{0}^{t^{*}}\left(\phi\left(\hat{Y}_{h}\right), \kappa\right) d t+\left(\left(Y_{h}-y\left(U_{h}\right)\right)(x, 0), \kappa(x, 0)\right) \\
& +\int_{0}^{t^{*}}\left(\phi\left(Y_{h}\right)-\phi\left(\hat{Y}_{h}\right), \kappa\right) d t \\
= & \int_{0}^{t^{*}}\left(Y_{h t}+\operatorname{div} \hat{P}_{h}+\phi\left(\hat{Y}_{h}\right)-\hat{f}-U_{h}, \kappa\right) d t \\
& \left.+\int_{0}^{t^{*}}(\hat{f}-f, \kappa)+\left(\hat{P}_{h}-P_{h}, \nabla \kappa\right)\right) d t
\end{aligned}
$$




$$
\begin{aligned}
& +\int_{0}^{t^{*}}\left(\nabla w_{h}-\alpha P_{h}, A \nabla \kappa-\Pi_{h}(A \nabla \kappa)\right) d t \\
& +\int_{0}^{t^{*}}\left(\phi\left(Y_{h}\right)-\phi\left(\hat{Y}_{h}\right), \kappa\right) d t+\left(\left(Y_{h}-y\left(U_{h}\right)\right)(x, 0), \kappa(x, 0)\right)
\end{aligned}
$$

When $t^{*} \in\left(t_{i-1}, t_{i}\right], i \leq 2$,

$$
\begin{aligned}
\left\|\left(Y_{h}-y\left(U_{h}\right)\right)\left(x, t^{*}\right)\right\|_{L^{2}(\Omega)}^{2} \\
\leq C \int_{t_{0}}^{t_{2}} \sum_{\tau} h_{\tau}^{2} \int_{\tau}\left(Y_{h t}+\operatorname{div} \hat{P}_{h}-\hat{f}-U_{h}\right)^{2} d x d t \\
\quad+C \int_{t_{0}}^{t_{2}} \sum_{\tau} \min _{w_{h} \in W_{h}} \int_{\tau}\left(\alpha P_{h}-\nabla w_{h}\right)^{2} d x d t \\
\quad+C\|\hat{f}-f\|_{L^{1}\left(0, t^{*} ; L^{2}(\Omega)\right)}^{2}+C\left\|\hat{P}_{h}-P_{h}\right\|_{L^{2}\left(0, t^{*} ; L^{2}(\Omega)\right)}^{2} \\
\quad+C\left\|\hat{Y}_{h}-Y_{h}\right\|_{L^{2}\left(0, t^{*} ; L^{2}(\Omega)\right)}^{2}+C\left\|y_{0}^{h}(x)-y_{0}(x)\right\|_{L^{2}(\Omega)}^{2}
\end{aligned}
$$

When $i>2$,

$$
\begin{aligned}
&\left\|\left(Y_{h}-y\left(U_{h}\right)\right)\left(x, t^{*}\right)\right\|_{L^{2}(\Omega)}^{2} \\
& \leq C \int_{t_{i-2}}^{t_{i}} \sum_{\tau} h_{\tau}^{2} \int_{\tau}\left(Y_{h t}+\operatorname{div} \hat{P}_{h}-\hat{f}-U_{h}\right)^{2} d x d t \\
&+C\left|\ln \frac{\Delta t}{t^{*}}\right| \max _{t \in\left[0, t_{i-2}\right]}\left\{\sum_{\tau} h_{\tau}^{4} \int_{\tau}\left(Y_{h t}+\operatorname{div} \hat{P}_{h}-\hat{f}-U_{h}\right)^{2} d x\right\} \\
&+C \int_{t_{i-2}}^{t_{i}} \sum_{\tau} \min _{w_{h} \in W_{h}} \int_{\tau}\left(\alpha P_{h}-\nabla w_{h}\right)^{2} d x d t \\
&+C \mid \ln \frac{\Delta t}{t^{*}} \max _{t \in\left[0, t_{i-2}\right]}\left\{\sum_{\tau} h_{\tau}^{2} \cdot \min _{w_{h} \in W_{h}} \int_{\tau}\left(\alpha P_{h}-\nabla w_{h}\right)^{2} d x\right\} \\
&+C\|\hat{f}-f\|_{L^{1}\left(0, t^{*} ; L^{2}(\Omega)\right)}^{2}+C\left\|\hat{P}_{h}-P_{h}\right\|_{L^{2}\left(0, t^{*} ; L^{2}(\Omega)\right)}^{2} \\
&+\left(\phi\left(Y_{h}\right)-\phi\left(\hat{Y}_{h}\right), \kappa\right)+C\left\|y_{0}^{h}(x)-y_{0}(x)\right\|_{L^{2}(\Omega)}^{2}
\end{aligned}
$$

Hence

$$
\left\|Y_{h}-y\left(U_{h}\right)\right\|_{L^{\infty}\left(j ; L^{2}(\Omega)\right)}^{2} \leq C \sum_{i=2}^{7} \eta_{i}^{2} .
$$

Similar to Theorem 3.2 of reference [16], we have derived the following estimate:

$$
\begin{aligned}
\left\|P_{h}-\mathbf{p}\left(U_{h}\right)\right\|_{L^{2}\left(j ; L^{2}(\Omega)\right)} & \\
\leq & C\left(\|\hat{f}-f\|_{L^{2}\left(J ; L^{2}(\Omega)\right)}+\left\|\left(\hat{Y}_{h}-Y_{h}\right)_{t}\right\|_{L^{2}\left(j ; L^{2}(\Omega)\right)}\right. \\
& \left.+\left\|\hat{P}_{h}-P_{h}\right\|_{L^{2}\left(j ; L^{2}(\Omega)\right)}+\left\|y_{0}^{h}(x)-y_{0}(x)\right\|_{L^{2}(\Omega)}^{2}\right) .
\end{aligned}
$$

This proves (3.19). 
Now, we are in a position to estimate the errors $Z_{h}-z\left(U_{h}\right)$ and $Q_{h}-\mathbf{q}\left(U_{h}\right)$.

Theorem 3.3 Let $\left(P_{h}, Y_{h}, Q_{h}, Z_{h}, U_{h}\right)$ and $\left(\mathbf{p}\left(U_{h}\right), y\left(U_{h}\right), \mathbf{q}\left(U_{h}\right), z\left(U_{h}\right), U_{h}\right)$ be the solutions of (2.38)-(2.44) and (2.46)-(2.51), respectively. Then we have the following error estimate:

$$
\left\|Z_{h}-z\left(U_{h}\right)\right\|_{L^{\infty}\left(j ; L^{2}(\Omega)\right)}^{2}+\left\|Q_{h}-\mathbf{q}\left(U_{h}\right)\right\|_{L^{2}\left(J ; L^{2}(\Omega)\right)} \leq C \sum_{i=2}^{15} \eta_{i}^{2}
$$

where $\eta_{2}-\eta_{7}$ are defined in Theorem 3.2, and

$$
\begin{aligned}
& \eta_{8}^{2}=\max _{i \in[1, N-1]}\left\{\int_{t_{i-1}}^{t_{i+1}} \sum_{\tau} h_{\tau}^{2} \int_{\tau}\left(-Z_{h t}+\operatorname{div} \widetilde{Q}_{h}+\phi^{\prime}\left(\hat{Y}_{h}\right) \widetilde{Z}_{h}-\widetilde{Y}_{h}+\tilde{y}_{d}\right)^{2} d x d t\right\} ; \\
& \eta_{9}^{2}=\max _{i \in[1, N-1]}\left\{\int_{t_{i-1}}^{t_{i+1}} \sum_{\tau} \min _{w_{h} \in W_{h}} \int_{\tau}\left(\alpha Q_{h}+P_{h}-\overline{\mathbf{p}}_{d}-\nabla w_{h}\right)^{2} d x d t\right\} ; \\
& \eta_{10}^{2}=|\ln \Delta t| \max _{t \in[0, T]}\left\{\sum_{\tau} h_{\tau}^{4} \int_{\tau}\left(-Z_{h t}+\operatorname{div} \widetilde{Q}_{h}+\phi^{\prime}\left(\hat{Y}_{h}\right) \widetilde{Z}_{h}-\widetilde{Y}_{h}+\tilde{y}_{d}\right)^{2} d x\right\} \\
& \eta_{11}^{2}=|\ln \Delta t| \max _{t \in[0, T]}\left\{\sum_{\tau} h_{\tau}^{2} \cdot \min _{w_{h} \in W_{h}} \int_{\tau}\left(\alpha Q_{h}+P_{h}-\overline{\mathbf{p}}_{d}-\nabla w_{h}\right)^{2} d x\right\} \\
& \eta_{12}^{2}=\left\|\widetilde{Q}_{h}-Q_{h}\right\|_{L^{2}\left(J ; L^{2}(\Omega)\right)}^{2}+\left\|\widetilde{P}_{h}-P_{h}\right\|_{L^{2}\left(j ; L^{2}(\Omega)\right)}^{2} ; \\
& \eta_{13}^{2}=\left\|\mathbf{p}_{d}-\tilde{\mathbf{p}}_{d}\right\|_{L^{2}\left(J ; L^{2}(\Omega)\right)}^{2}+\left\|\overline{\mathbf{p}}_{d}-\mathbf{p}_{d}\right\|_{L^{2}\left(J ; L^{2}(\Omega)\right)^{2}}^{2} \\
& \eta_{14}^{2}=\left\|\widetilde{Y}_{h}-Y_{h}\right\|_{L^{2}\left(J ; L^{2}(\Omega)\right)}^{2}+\left\|\tilde{y}_{d}-y_{d}\right\|_{L^{2}\left(J ; L^{2}(\Omega)\right)}^{2} ; \\
& \eta_{15}^{2}=\left\|\widetilde{Z}_{h}-Z_{h}\right\|_{L^{2}\left(J ; L^{2}(\Omega)\right)}^{2}+\left\|\left(\widetilde{Z}_{h}-Z_{h}\right)_{t}\right\|_{L^{2}\left(J ; L^{2}(\Omega)\right)}^{2}
\end{aligned}
$$

Proof We first define $\mathbf{q}_{h}^{N}$ as follows:

$$
\left(\alpha \mathbf{q}_{h}^{N}, \mathbf{v}_{h}\right)-\left(z_{h}^{N}, \operatorname{div} \mathbf{v}_{h}\right)=-\left(\mathbf{p}_{h}^{N}-\mathbf{p}_{d}^{N}, \mathbf{v}_{h}\right), \quad \forall \mathbf{v}_{h} \in \mathbf{V}_{h}
$$

Then from (2.34) and (3.29) we deduce that

$$
\left(\alpha \hat{Q}_{h}, \mathbf{v}_{h}\right)-\left(\hat{Z}_{h}, \operatorname{div} \mathbf{v}_{h}\right)=-\left(\hat{P}_{h}-\hat{\mathbf{p}}_{d}, \mathbf{v}_{h}\right), \quad \forall \mathbf{v}_{h} \in \mathbf{V}_{h}
$$

Now, we let

$$
\left.\overline{\mathbf{p}}_{d}\right|_{\left(t_{i-1}, t_{i}\right]}=\left(\left(t_{i}-t\right) \mathbf{p}_{d}^{i-1}+\left(t-t_{i-1}\right) \mathbf{p}_{d}^{i}\right) / \Delta t
$$

Combining (2.41), (3.30) and the definitions of $Z_{h}, Q_{h}, P_{h}$ and $\overline{\mathbf{p}}_{d}$, we get

$$
\left(\alpha Q_{h}, \mathbf{v}_{h}\right)-\left(Z_{h}, \operatorname{div} \mathbf{v}_{h}\right)=-\left(P_{h}-\overline{\mathbf{p}}_{d}, \mathbf{v}_{h}\right), \quad \forall \mathbf{v}_{h} \in \mathbf{V}_{h}
$$

Let $\varpi$ be the solution of (3.17) with $\varpi_{0}(x)=\left(Z_{h}-z\left(U_{h}\right)\right)\left(x, t^{*}\right)$. Then it follows from (2.41)-(2.43), (2.49)-(2.51) and (2.56)-(2.58) that 


$$
\begin{aligned}
& \left\|\left(Z_{h}-z\left(U_{h}\right)\right)\left(x, t^{*}\right)\right\|_{L^{2}(\Omega)}^{2} \\
& =\left(\left(Z_{h}-z\left(U_{h}\right)\right)\left(x, t^{*}\right), \varpi\left(x, t^{*}\right)\right) \\
& =\int_{t^{*}}^{T}\left(-\left(\left(Z_{h}-z\left(U_{h}\right)\right)_{t}, \varpi\right)-\left(Z_{h}-z\left(U_{h}\right), \operatorname{div}\left(A^{*} \nabla \varpi\right)\right)\right. \\
& \left.+\left(\phi^{\prime}\left(y\left(U_{h}\right)\right)\left(Z_{h}-z\left(U_{h}\right)\right), \varpi\right)\right) d t \\
& =\int_{t^{*}}^{T}\left(-\left(\left(Z_{h}-z\left(U_{h}\right)\right)_{t^{\prime}},\right)+\left(\mathbf{q}\left(U_{h}\right), \nabla \varpi\right)+\left(\phi^{\prime}\left(\hat{Y}_{h}\right) \widetilde{Z}_{h}, \varpi\right)\right) d t \\
& +\int_{t^{*}}^{T}\left(\left(\mathbf{p}\left(U_{h}\right)-\mathbf{p}_{d}, \nabla \varpi\right)-\left(Z_{h}, \operatorname{div}(A \nabla \varpi)\right)\right) d t \\
& +\int_{t^{*}}^{T}\left(\left(\phi^{\prime}\left(y\left(U_{h}\right)\right)\left(Z_{h}-\widetilde{Z}_{h}\right), \varpi\right)+\left(\left(\phi^{\prime}\left(y\left(U_{h}\right)\right)-\phi^{\prime}\left(\hat{Y}_{h}\right)\right) \widetilde{Z}_{h}, \varpi\right)\right) d t \\
& =\int_{t^{*}}^{T}\left(-\left(\left(Z_{h}-z\left(U_{h}\right)\right)_{t}, \varpi\right)+\left(\operatorname{div}\left(\widetilde{Q}_{h}-\mathbf{q}\left(U_{h}\right)\right), \varpi\right)+\left(\phi^{\prime}\left(\hat{Y}_{h}\right) \widetilde{Z}_{h}, \varpi\right)\right) d t \\
& +\int_{t^{*}}^{T}\left(\left(\mathbf{p}\left(U_{h}\right)-\mathbf{p}_{d}, \nabla \varpi\right)-\left(\operatorname{div} \widetilde{Q}_{h}, \varpi\right)\right) d t \\
& -\int_{t^{*}}^{T}\left(Z_{h}, \operatorname{div}\left(\Pi_{h}(A \nabla \varpi)\right)\right) d t+\int_{t^{*}}^{T}\left(\phi^{\prime}\left(y\left(U_{h}\right)\right)\left(Z_{h}-\widetilde{Z}_{h}\right), \varpi\right) d t \\
& +\int_{t^{*}}^{T}\left(\tilde{\phi}^{\prime \prime}\left(y\left(U_{h}\right)\right)\left(y\left(U_{h}\right)-\hat{Y}_{h}\right) \widetilde{Z}_{h}, \varpi\right) d t \\
& =\int_{t^{*}}^{T}\left(-Z_{h t}+\operatorname{div} \widetilde{Q}_{h}+\phi^{\prime}\left(\hat{Y}_{h}\right) \widetilde{Z}_{h}-\widetilde{Y}_{h}+\tilde{y}_{d}, \varpi\right) d t \\
& -\int_{t^{*}}^{T}\left(y\left(U_{h}\right)-y_{d}-\tilde{Y}_{h}+\tilde{y}_{d}, \varpi\right) d t \\
& +\int_{t^{*}}^{T}\left(\left(\mathbf{p}\left(U_{h}\right)-\mathbf{p}_{d}, \nabla \varpi\right)+\left(\widetilde{Q}_{h}, \nabla \varpi\right)\right) d t \\
& -\int_{t^{*}}^{T}\left(\alpha Q_{h}+P_{h}-\overline{\mathbf{p}}_{d}, \Pi_{h}(A \nabla \varpi)\right) d t \\
& +\int_{t^{*}}^{T}\left(\left(\phi^{\prime}\left(y\left(U_{h}\right)\right)\left(Z_{h}-\widetilde{Z}_{h}\right), \varpi\right)+\left(\tilde{\phi}^{\prime \prime}\left(y\left(U_{h}\right)\right)\left(y\left(U_{h}\right)-\hat{Y}_{h}\right) \widetilde{Z}_{h}, \varpi\right)\right) d t \\
& =\int_{t^{*}}^{T}\left(-Z_{h t}+\operatorname{div} \widetilde{Q}_{h}+\phi^{\prime}\left(\hat{Y}_{h}\right) \widetilde{Z}_{h}-\widetilde{Y}_{h}+\tilde{y}_{d}, \varpi\right) d t \\
& +\int_{t^{*}}^{T}\left(\alpha Q_{h}+P_{h}-\overline{\mathbf{p}}_{d}-\nabla w_{h}, A \nabla \varpi-\Pi_{h}(A \nabla \varpi)\right) d t \\
& +\int_{t^{*}}^{T}\left(\mathbf{p}\left(U_{h}\right)-P_{h}+\overline{\mathbf{p}}_{d}-\mathbf{p}_{d}+\widetilde{Q}_{h}-Q_{h}, \nabla \varpi\right) d t \\
& +\int_{t^{*}}^{T}\left(\tilde{y}_{d}-y_{d}+\tilde{Y}_{h}-y\left(U_{h}\right), \varpi\right) d t \\
& +\int_{t^{*}}^{T}\left(\phi^{\prime}\left(y\left(U_{h}\right)\right)\left(Z_{h}-\widetilde{Z}_{h}\right), \varpi\right) d t+\int_{t^{*}}^{T}\left(\tilde{\phi}^{\prime \prime}\left(y\left(U_{h}\right)\right)\left(y\left(U_{h}\right)-\hat{Y}_{h}\right) \widetilde{Z}_{h}, \varpi\right) d t \\
& \equiv E_{1}+E_{2}+E_{3}+E_{4}+E_{5}+E_{6} .
\end{aligned}
$$


To prove (3.28), the first step is to estimate $E_{1}$. Let $t^{*} \in\left(t_{i-1}, t_{i}\right]$, when $i \geq N-1$, by Lemmas 2.1, 2.2 and 3.1, we have

$$
\begin{aligned}
E_{1}= & \int_{t^{*}}^{T}\left(-Z_{h t}+\operatorname{div} \widetilde{Q}_{h}+\phi^{\prime}\left(\hat{Y}_{h}\right) \widetilde{Z}_{h}-\widetilde{Y}_{h}+\tilde{y}_{d}, \varpi-\hat{\pi}_{h} \varpi\right) d t \\
\leq & C \int_{t^{*}}^{T} \sum_{\tau}\left\|-Z_{h t}+\operatorname{div} \widetilde{Q}_{h}+\phi^{\prime}\left(\hat{Y}_{h}\right) \widetilde{Z}_{h}-\widetilde{Y}_{h}+\tilde{y}_{d}\right\|_{L^{2}(\tau)} h_{\tau}|\varpi|_{H^{1}(\tau)} d t \\
\leq & C(\delta) \int_{t^{*}}^{T} \sum_{\tau} h_{\tau}^{2} \int_{\tau}\left(-Z_{h t}+\operatorname{div} \widetilde{Q}_{h}+\phi^{\prime}\left(\hat{Y}_{h}\right) \widetilde{Z}_{h}-\widetilde{Y}_{h}+\tilde{y}_{d}\right)^{2} d x d t \\
& +C \delta \int_{t^{*}}^{T} \int_{\Omega}|\nabla \varpi|^{2} d x d t \\
\leq & C(\delta) \int_{t_{N-2}}^{t_{N}} \sum_{\tau} h_{\tau}^{2} \int_{\tau}\left(-Z_{h t}+\operatorname{div} \widetilde{Q}_{h}+\phi^{\prime}\left(\hat{Y}_{h}\right) \widetilde{Z}_{h}-\widetilde{Y}_{h}+\tilde{y}_{d}\right)^{2} d x d t \\
& +C \delta\left\|\left(Z_{h}-z\left(U_{h}\right)\right)\left(x, t^{*}\right)\right\|_{L^{2}(\Omega)}^{2} .
\end{aligned}
$$

When $i<N-1$,

$$
\begin{aligned}
E_{1}= & \int_{t^{*}}^{t_{i+1}}\left(-Z_{h t}+\operatorname{div} \widetilde{Q}_{h}+\phi^{\prime}\left(\hat{Y}_{h}\right) \widetilde{Z}_{h}-\widetilde{Y}_{h}+\tilde{y}_{d}, \varpi-\hat{\pi}_{h} \varpi\right) d t \\
& +\int_{t_{i+1}}^{T}\left(-Z_{h t}+\operatorname{div} \widetilde{Q}_{h}+\phi^{\prime}\left(\hat{Y}_{h}\right) \widetilde{Z}_{h}-\widetilde{Y}_{h}+\tilde{y}_{d}, \varpi-\pi_{h} \varpi\right) d t \\
\leq & C \int_{t^{*}}^{t_{i+1}} \sum_{\tau}\left\|-Z_{h t}+\operatorname{div} \widetilde{Q}_{h}+\phi^{\prime}\left(\hat{Y}_{h}\right) \widetilde{Z}_{h}-\widetilde{Y}_{h}+\tilde{y}_{d}\right\|_{L^{2}(\tau)} h_{\tau}|\varpi|_{H^{1}(\tau)} d t \\
& +C \int_{t_{i+1}}^{T} \sum_{\tau}\left\|-Z_{h t}+\operatorname{div} \widetilde{Q}_{h}+\phi^{\prime}\left(\hat{Y}_{h}\right) \widetilde{Z}_{h}-\widetilde{Y}_{h}+\tilde{y}_{d}\right\|_{L^{2}(\tau)} h_{\tau}^{2}|\varpi|_{H^{2}(\tau)} d t \\
\leq & C(\delta) \int_{t^{*}}^{t_{i+1}} \sum_{\tau} h_{\tau}^{2} \int_{\tau}\left(-Z_{h t}+\operatorname{div} \widetilde{Q}_{h}+\phi^{\prime}\left(\hat{Y}_{h}\right) \widetilde{Z}_{h}-\widetilde{Y}_{h}+\tilde{y}_{d}\right)^{2} d x d t \\
& +C(\delta) \int_{t_{i+1}}^{T}\left|t-t^{*}\right|^{-1} \sum_{\tau} h_{\tau}^{4} \int_{\tau}\left(-Z_{h t}+\operatorname{div} \widetilde{Q}_{h}+\phi^{\prime}\left(\hat{Y}_{h}\right) \widetilde{Z}_{h}-\widetilde{Y}_{h}+\tilde{y}_{d}\right)^{2} d x d t \\
& +C \delta \int_{t^{*}}^{t_{i+1}} \int_{\Omega}|\nabla \varpi|^{2} d x d t+C \delta \int_{t_{i+1}}^{T}\left|t-t^{*}\right| \int_{\Omega}\left|D^{2} \varpi\right|^{2} d x d t \\
\leq & C(\delta) \int_{t_{i-1}}^{t_{i+1}} \sum_{\tau} h_{\tau}^{2} \int_{\tau}\left(-Z_{h t}+\operatorname{div} \widetilde{Q}_{h}+\phi^{\prime}\left(\hat{Y}_{h}\right) \widetilde{Z}_{h}-\widetilde{Y}_{h}+\tilde{y}_{d}\right)^{2} d x d t \\
& +C(\delta) \mid \ln \frac{\Delta t}{T-t^{*} \mid} \\
& \times \max _{t \in\left[t_{i+1}, T\right]}\left\{\sum_{\tau} h_{\tau}^{4} \int_{\tau}\left(-Z_{h t}+\operatorname{div} \widetilde{Q}_{h}+\phi^{\prime}\left(\hat{Y}_{h}\right) \widetilde{Z}_{h}-\widetilde{Y}_{h}+\tilde{y}_{d}\right)^{2} d x\right\} \\
& +C \delta\left\|\left(Z_{h}-z\left(U_{h}\right)\right)\left(x, t^{*}\right)\right\|_{L^{2}(\Omega)}^{2} \cdot
\end{aligned}
$$


Now we estimate $E_{2}$. Let $t^{*} \in\left(t_{i-1}, t_{i}\right]$ again. Similarly, when $i \geq N-1$,

$$
\begin{aligned}
E_{2}= & \int_{t_{*}}^{T}\left(\alpha Q_{h}+P_{h}-\overline{\mathbf{p}}_{d}-\nabla w_{h}, A \nabla \varpi-\Pi_{h}(A \nabla \varpi)\right) d t \\
\leq & C(\delta) \int_{t_{N-2}}^{t_{N}} \sum_{\tau} \min _{w_{h} \in W_{h}} \int_{\tau}\left(\alpha Q_{h}+P_{h}-\overline{\mathbf{p}}_{d}-\nabla w_{h}\right)^{2} d x d t \\
& +C \delta\left\|\left(Z_{h}-z\left(U_{h}\right)\right)\left(x, t^{*}\right)\right\|_{L^{2}(\Omega)}^{2} .
\end{aligned}
$$

When $i<N-1$,

$$
\begin{aligned}
E_{2} \leq & C(\delta) \int_{t_{i-1}}^{t_{i+1}} \sum_{\tau} \min _{w_{h} \in W_{h}} \int_{\tau}\left(\alpha Q_{h}+P_{h}-\overline{\mathbf{p}}_{d}-\nabla w_{h}\right)^{2} d x d t \\
& +C(\delta)\left|\ln \frac{\Delta t}{T-t^{*}}\right| \max _{t \in\left[t_{i+1}, T\right]}\left\{\sum_{\tau} h_{\tau}^{2} \cdot \min _{w_{h} \in W_{h}} \int_{\tau}\left(\alpha Q_{h}+P_{h}-\overline{\mathbf{p}}_{d}-\nabla w_{h}\right)^{2} d x\right\} \\
& +C \delta\left\|\left(Z_{h}-z\left(U_{h}\right)\right)\left(x, t^{*}\right)\right\|_{L^{2}(\Omega)}^{2} .
\end{aligned}
$$

Next we estimate $E_{3}, E_{4}$. It follows from Lemma 3.1 that

$$
\begin{aligned}
E_{3}= & \int_{t^{*}}^{T}\left(\mathbf{p}\left(U_{h}\right)-P_{h}+\overline{\mathbf{p}}_{d}-\mathbf{p}_{d}+\widetilde{Q}_{h}-Q_{h}, \nabla \varpi\right) d t \\
\leq & C(\delta)\left\|P_{h}-\mathbf{p}\left(U_{h}\right)\right\|_{L^{2}\left(t^{*}, T ; L^{2}(\Omega)\right)}^{2}+C(\delta)\left\|\overline{\mathbf{p}}_{d}-\mathbf{p}_{d}\right\|_{L^{2}\left(t^{*}, T ; L^{2}(\Omega)\right)}^{2} \\
& +C(\delta)\left\|\widetilde{Q}_{h}-Q_{h}\right\|_{L^{2}\left(t^{*}, T ; L^{2}(\Omega)\right)}^{2}+C \delta \int_{t^{*}}^{T} \int_{\Omega}|\nabla \varpi|^{2} d x d t \\
\leq & C(\delta)\left\|P_{h}-\mathbf{p}\left(U_{h}\right)\right\|_{L^{2}\left(t^{*}, T ; L^{2}(\Omega)\right)}^{2}+C(\delta)\left\|\overline{\mathbf{p}}_{d}-\mathbf{p}_{d}\right\|_{L^{2}\left(t^{*}, T ; L^{2}(\Omega)\right)}^{2} \\
& +C(\delta)\left\|\widetilde{Q}_{h}-Q_{h}\right\|_{L^{2}\left(t^{*}, T ; L^{2}(\Omega)\right)}^{2}+C \delta\left\|\left(Z_{h}-z\left(U_{h}\right)\right)\left(x, t^{*}\right)\right\|_{L^{2}(\Omega)}^{2},
\end{aligned}
$$

and

$$
\begin{aligned}
E_{4}= & \int_{t^{*}}^{T}\left(\tilde{y}_{d}-y_{d}+\tilde{Y}_{h}-y\left(U_{h}\right), \varpi\right) d t \\
\leq & C(\delta)\left\|\tilde{y}_{d}-y_{d}\right\|_{L^{1}\left(t^{*}, T ; L^{2}(\Omega)\right)}^{2}+C(\delta)\left\|\tilde{Y}_{h}-y\left(U_{h}\right)\right\|_{L^{1}\left(t^{*}, T ; L^{2}(\Omega)\right)}^{2} \\
& +C \delta \max _{t \in\left[t^{*}, T\right]}\left\{\|\varpi(x, t)\|_{L^{2}(\Omega)}^{2}\right\} \\
\leq & C(\delta)\left\|\tilde{y}_{d}-y_{d}\right\|_{L^{1}\left(t^{*}, T ; L^{2}(\Omega)\right)}^{2}+C(\delta)\left\|\tilde{Y}_{h}-Y_{h}\right\|_{L^{1}\left(t^{*}, T ; L^{2}(\Omega)\right)}^{2} \\
& +C(\delta)\left\|Y_{h}-y\left(U_{h}\right)\right\|_{L^{1}\left(t^{*}, T ; L^{2}(\Omega)\right)}^{2}+C \delta\left\|\left(Z_{h}-z\left(U_{h}\right)\right)\left(x, t^{*}\right)\right\|_{L^{2}(\Omega)}^{2}
\end{aligned}
$$

Furthermore, we estimate $E_{5}, E_{6}$. It follows from Lemma 3.1 that

$$
\begin{aligned}
E_{5} & =\int_{t^{*}}^{T}\left(\phi^{\prime}\left(y\left(U_{h}\right)\right)\left(Z_{h}-\widetilde{Z}_{h}\right), \varpi\right) d t \\
& \leq C(\delta)\left\|\widetilde{Z}_{h}-Z_{h}\right\|_{L^{2}\left(t^{*}, T ; L^{2}(\Omega)\right)}^{2}+C \delta \max _{t \in\left[t^{*}, T\right]}\left\{\|\varpi(x, t)\|_{L^{2}(\Omega)}^{2}\right\} \\
& \leq C(\delta)\left\|\widetilde{Z}_{h}-Z_{h}\right\|_{L^{2}\left(t^{*}, T ; L^{2}(\Omega)\right)}^{2}+C \delta\left\|\left(Z_{h}-z\left(U_{h}\right)\right)\left(x, t^{*}\right)\right\|_{L^{2}(\Omega)}^{2},
\end{aligned}
$$


and

$$
\begin{aligned}
E_{6}= & \int_{t^{*}}^{T}\left(\tilde{\phi}^{\prime \prime}\left(y\left(U_{h}\right)\right)\left(y\left(U_{h}\right)-\hat{Y}_{h}\right) \widetilde{Z}_{h}, \varpi\right) d t \\
\leq & C(\delta)\left\|\hat{Y}_{h}-y\left(U_{h}\right)\right\|_{L^{1}\left(t^{*}, T ; L^{2}(\Omega)\right)}^{2}+C \delta \max _{t \in\left[t^{*}, T\right]}\left\{\|\varpi(x, t)\|_{L^{2}(\Omega)}^{2}\right\} \\
\leq & C(\delta)\left\|Y_{h}-y\left(U_{h}\right)\right\|_{L^{1}\left(t^{*}, T ; L^{2}(\Omega)\right)}^{2}+C(\delta)\left\|\widetilde{Y}_{h}-Y_{h}\right\|_{L^{1}\left(t^{*}, T ; L^{2}(\Omega)\right)}^{2} \\
& +C \delta\left\|\left(Z_{h}-z\left(U_{h}\right)\right)\left(x, t^{*}\right)\right\|_{L^{2}(\Omega)}^{2} .
\end{aligned}
$$

Hence, from (3.33)-(3.40) we have that when $t^{*} \in\left(t_{i-1}, t_{i}\right], i \geq N-1$,

$$
\begin{aligned}
\left\|\left(Z_{h}-z\left(U_{h}\right)\right)\left(x, t^{*}\right)\right\|_{L^{2}(\Omega)}^{2} \\
\leq C \int_{t_{N-2}}^{t_{N}} \sum_{\tau} h_{\tau}^{2} \int_{\tau}\left(-Z_{h t}+\operatorname{div} \widetilde{Q}_{h}+\phi^{\prime}\left(\hat{Y}_{h}\right) \widetilde{Z}_{h}-\widetilde{Y}_{h}+\tilde{y}_{d}\right)^{2} d x d t \\
\quad+C \int_{t_{N-2}}^{t_{N}} \sum_{\tau} \min _{w_{h} \in W_{h}} \int_{\tau}\left(\alpha Q_{h}+P_{h}-\overline{\mathbf{p}}_{d}-\nabla w_{h}\right)^{2} d x d t \\
\quad+C\left\|Y_{h}-y\left(U_{h}\right)\right\|_{L^{1}\left(t^{*}, T ; L^{2}(\Omega)\right)}^{2}+C\left\|P_{h}-\mathbf{p}\left(U_{h}\right)\right\|_{L^{2}\left(t^{*}, T ; L^{2}(\Omega)\right)}^{2} \\
\quad+C\left\|\widetilde{Q}_{h}-Q_{h}\right\|_{L^{2}\left(t^{*}, T ; L^{2}(\Omega)\right)}^{2}+C\left\|\widetilde{Y}_{h}-Y_{h}\right\|_{L^{1}\left(t^{*}, T ; L^{2}(\Omega)\right)}^{2} \\
\quad+C\left\|\widetilde{Z}_{h}-Z_{h}\right\|_{L^{2}\left(t^{*}, T ; L^{2}(\Omega)\right)}^{2}+C\left\|\tilde{y}_{d}-y_{d}\right\|_{L^{1}\left(t^{*}, T ; L^{2}(\Omega)\right)}^{2} \\
\quad+C\left\|\overline{\mathbf{p}}_{d}-\mathbf{p}_{d}\right\|_{L^{2}\left(t^{*}, T ; L^{2}(\Omega)\right)}^{2} .
\end{aligned}
$$

When $i<N-1$,

$$
\begin{aligned}
&\left\|\left(Z_{h}-z\left(U_{h}\right)\right)\left(x, t^{*}\right)\right\|_{L^{2}(\Omega)}^{2} \\
& \leq C \int_{t_{i-1}}^{t_{i+1}} \sum_{\tau} h_{\tau}^{2} \int_{\tau}\left(-Z_{h t}+\operatorname{div} \widetilde{Q}_{h}-\widetilde{Y}_{h}+\tilde{y}_{d}\right)^{2} d x d t \\
&+C\left|\ln \frac{\Delta t}{T-t^{*}}\right| \max _{t \in\left[t_{i+1}, T\right]}\left\{\sum_{\tau} h_{\tau}^{4} \int_{\tau}\left(-Z_{h t}+\operatorname{div} \widetilde{Q}_{h}+\phi^{\prime}\left(\hat{Y}_{h}\right) \widetilde{Z}_{h}-\widetilde{Y}_{h}+\tilde{y}_{d}\right)^{2} d x\right\} \\
&+C \int_{t_{i-1}}^{t_{i+1}} \sum_{\tau} \min _{w_{h} \in W_{h}} \int_{\tau}\left(\alpha Q_{h}+P_{h}-\overline{\mathbf{p}}_{d}-\nabla w_{h}\right)^{2} d x d t \\
&+C\left|\ln \frac{\Delta t}{T-t^{*}}\right|_{t \in\left[t_{i+1}, T\right]}\left\{\sum_{\tau} h_{\tau}^{2} \cdot \min _{w_{h} \in W_{h}} \int_{\tau}\left(\alpha Q_{h}+P_{h}-\overline{\mathbf{p}}_{d}-\nabla w_{h}\right)^{2} d x\right\} \\
&+C\left\|Y_{h}-y\left(U_{h}\right)\right\|_{L^{1}\left(t^{*}, T ; L^{2}(\Omega)\right)}^{2}+C\left\|P_{h}-\mathbf{p}\left(U_{h}\right)\right\|_{L^{2}\left(t^{*}, T ; L^{2}(\Omega)\right)}^{2} \\
&+C\left\|\widetilde{Q}_{h}-Q_{h}\right\|_{L^{2}\left(t^{*}, T ; L^{2}(\Omega)\right)}^{2}+C\left\|\tilde{Y}_{h}-Y_{h}\right\|_{L^{1}\left(t^{*}, T ; L^{2}(\Omega)\right)}^{2} \\
&+C\left\|\widetilde{Z}_{h}-Z_{h}\right\|_{L^{2}\left(t^{*}, T ; L^{2}(\Omega)\right)}^{2}+C\left\|\tilde{y}_{d}-y_{d}\right\|_{L^{1}\left(t^{*}, T ; L^{2}(\Omega)\right)}^{2} \\
&+C\left\|\overline{\mathbf{p}}_{d}-\mathbf{p}_{d}\right\|_{L^{2}\left(t^{*}, T ; L^{2}(\Omega)\right)}^{2}
\end{aligned}
$$


Then it follows from (3.41)-(3.42) that

$$
\begin{aligned}
\left\|Z_{h}-z\left(U_{h}\right)\right\|_{L^{\infty}\left(j ; L^{2}(\Omega)\right)}^{2} \leq & C \sum_{i=8}^{15} \eta_{i}^{2}+C\left\|P_{h}-\mathbf{p}\left(U_{h}\right)\right\|_{L^{2}\left(j ; L^{2}(\Omega)\right)}^{2} \\
& +C\left\|Y_{h}-y\left(U_{h}\right)\right\|_{L^{2}\left(j ; L^{2}(\Omega)\right)^{2}}^{2}
\end{aligned}
$$

Similar to (3.27), we can prove that

$$
\begin{aligned}
\| Q_{h}- & \mathbf{q}\left(U_{h}\right) \|_{L^{2}\left(j ; L^{2}(\Omega)\right)} \\
\leq & C\left(\left\|Y_{h}-y\left(U_{h}\right)\right\|_{L^{2}\left(j ; L^{2}(\Omega)\right)}+\left\|P_{h}-\mathbf{p}\left(U_{h}\right)\right\|_{L^{2}\left(j ; L^{2}(\Omega)\right)}+\left\|\tilde{\mathbf{p}}_{d}-\mathbf{p}_{d}\right\|_{L^{2}\left(j L^{2}(\Omega)\right)}\right. \\
& +\left\|\widetilde{Q}_{h}-Q_{h}\right\|_{L^{2}\left(j ; L^{2}(\Omega)\right)}+\left\|\tilde{y}_{d}-y_{d}\right\|_{L^{2}\left(j ; L^{2}(\Omega)\right)} \\
& +\left\|\widetilde{Y}_{h}-Y_{h}\right\|_{L^{2}\left(j ; L^{2}(\Omega)\right)}+\left\|\widetilde{Z}_{h}-Z_{h}\right\|_{L^{2}\left(J ; L^{2}(\Omega)\right)} \\
& \left.+\left\|\left(\widetilde{Z}_{h}-Z_{h}\right)_{t}\right\|_{L^{2}\left(j ; L^{2}(\Omega)\right)}+\left\|\widetilde{P}_{h}-P_{h}\right\|_{L^{2}\left(j ; L^{2}(\Omega)\right)}\right) .
\end{aligned}
$$

The triangle inequality and (3.43) yield (3.28).

Let (p, $y, \mathbf{q}, z, u)$ and $\left(P_{h}, Y_{h}, Q_{h}, Z_{h}, U_{h}\right)$ be the solutions of (2.5)-(2.11) and (2.38)-(2.44), respectively. We decompose the errors as follows:

$$
\begin{aligned}
& \mathbf{p}-P_{h}=\mathbf{p}-\mathbf{p}\left(U_{h}\right)+\mathbf{p}\left(U_{h}\right)-P_{h}:=\epsilon_{1}+\varepsilon_{1}, \\
& y-Y_{h}=y-y\left(U_{h}\right)+y\left(U_{h}\right)-Y_{h}:=r_{1}+e_{1}, \\
& \mathbf{q}-Q_{h}=\mathbf{q}-\mathbf{q}\left(U_{h}\right)+\mathbf{q}\left(U_{h}\right)-Q_{h}:=\epsilon_{2}+\varepsilon_{2}, \\
& z-Z_{h}=z-z\left(U_{h}\right)+z\left(U_{h}\right)-Z_{h}:=r_{2}+e_{2} .
\end{aligned}
$$

From (2.5)-(2.11) and (2.38)-(2.44), we derive the error equations:

$$
\begin{aligned}
& \left(\alpha \epsilon_{1}, \mathbf{v}\right)-\left(r_{1}, \operatorname{div} \mathbf{v}\right)=0, \\
& \left(r_{1 t}, w\right)+\left(\operatorname{div} \epsilon_{1}, w\right)+\left(\phi(y)-\phi\left(y\left(U_{h}\right)\right), w\right)=\left(u-U_{h}, w\right), \\
& \left(\alpha \epsilon_{2}, \mathbf{v}\right)-\left(r_{2}, \operatorname{div} \mathbf{v}\right)=-\left(\epsilon_{1}, \mathbf{v}\right), \\
& \left(r_{2 t}, w\right)+\left(\operatorname{div} \epsilon_{2}, w\right)+\left(\phi^{\prime}(y) z-\phi^{\prime}\left(y\left(U_{h}\right)\right) z\left(U_{h}\right), w\right)=\left(r_{1}, w\right)
\end{aligned}
$$

for any $\mathbf{v} \in \mathbf{V}, w \in W$.

Theorem 3.4 Let $(\mathbf{p}, y, \mathbf{q}, z, u)$ and $\left(\mathbf{p}\left(U_{h}\right), y\left(U_{h}\right), \mathbf{q}\left(U_{h}\right), z\left(U_{h}\right), U_{h}\right)$ be the solutions of $(2.5)$ (2.11) and (2.46)-(2.51), respectively. There is a constant $C>0$, independent of $h$, such that

$$
\begin{aligned}
& \left\|\epsilon_{1}\right\|_{L^{2}\left(j ; L^{2}(\Omega)\right)}+\left\|r_{1}\right\|_{L^{\infty}\left(j ; L^{2}(\Omega)\right)} \leq C\left\|u-U_{h}\right\|_{L^{2}\left(j ; L^{2}(\Omega)\right)}, \\
& \left\|\epsilon_{2}\right\|_{L^{2}\left(j L^{2}(\Omega)\right)}+\left\|r_{2}\right\|_{L^{\infty}\left(; L^{2}(\Omega)\right)} \leq C\left\|u-U_{h}\right\|_{L^{2}\left(j L^{2}(\Omega)\right)} .
\end{aligned}
$$


Proof Part I. Choosing $\mathbf{v}=\epsilon_{1}$ and $w=r_{1}$ as the test functions and adding the two relations of (3.45)-(3.46), we have

$$
\begin{aligned}
\left(\alpha \epsilon_{1}, \epsilon_{1}\right)+\left(r_{1 t}, r_{1}\right) & =\left(u-U_{h}, r_{1}\right)-\left(\phi(y)-\phi\left(y\left(U_{h}\right)\right), r_{1}\right) \\
& =\left(u-U_{h}, r_{1}\right)-\left(\tilde{\phi}^{\prime}(y)\left(y-y\left(U_{h}\right)\right), r_{1}\right) .
\end{aligned}
$$

Then, using the $\epsilon$-Cauchy inequality, we find an estimate as follows:

$$
\left(\alpha \epsilon_{1}, \epsilon_{1}\right)+\left(r_{1 t}, r_{1}\right) \leq C\left(\left\|r_{1}\right\|_{L^{2}(\Omega)}^{2}+\left\|u-U_{h}\right\|_{L^{2}(\Omega)}^{2}\right)
$$

Note that

$$
\left(r_{1 t}, r_{1}\right)=\frac{1}{2} \frac{\partial}{\partial t}\left\|r_{1}\right\|_{L^{2}(\Omega)}^{2}
$$

then, using the assumption on $A$, we obtain that

$$
\left\|\epsilon_{1}\right\|_{L^{2}(\Omega)}^{2}+\frac{1}{2} \frac{\partial}{\partial t}\left\|r_{1}\right\|_{L^{2}(\Omega)}^{2} \leq C\left(\left\|r_{1}\right\|_{L^{2}(\Omega)}^{2}+\left\|u-U_{h}\right\|_{L^{2}(\Omega)}^{2}\right)
$$

Integrating (3.53) in time and since $r_{1}(0)=0$, applying Gronwall's lemma, we easily obtain the following error estimate:

$$
\left\|\epsilon_{1}\right\|_{L^{2}\left(j ; L^{2}(\Omega)\right)}^{2}+\left\|r_{1}\right\|_{L^{\infty}\left(j ; L^{2}(\Omega)\right)}^{2} \leq C\left\|u-U_{h}\right\|_{L^{2}\left(J ; L^{2}(\Omega)\right)}^{2} .
$$

This implies (3.49).

Part II. Similarly, choosing $v=\epsilon_{2}$ and $w=r_{2}$ as the test functions and adding the two relations of (3.47)-(3.48), we obtain that

$$
\begin{aligned}
\left(\alpha \epsilon_{2}, \epsilon_{2}\right)-\left(r_{2 t}, r_{2}\right)= & \left(g_{2}^{\prime}(y)-g_{2}^{\prime}\left(y\left(U_{h}\right)\right), r_{2}\right)-\left(g_{1}^{\prime}(\mathbf{p})-g_{1}^{\prime}\left(\mathbf{p}\left(U_{h}\right)\right), \epsilon_{2}\right) \\
& -\left(\phi^{\prime}(y) z-\phi^{\prime}\left(y\left(U_{h}\right)\right) z\left(U_{h}\right), r_{2}\right) .
\end{aligned}
$$

Then, using the $\epsilon$-Cauchy inequality, we find an estimate as follows:

$$
\left(\alpha \epsilon_{2}, \epsilon_{2}\right)+\left(r_{2 t}, r_{2}\right) \leq C\left(\left\|r_{1}\right\|_{L^{2}(\Omega)}^{2}+\left\|r_{2}\right\|_{L^{2}(\Omega)}^{2}+\left\|\epsilon_{1}\right\|_{L^{2}(\Omega)}^{2}\right)+\frac{c}{2}\left\|\epsilon_{2}\right\|_{L^{2}(\Omega)}^{2} .
$$

Note that

$$
\left(r_{2 t}, r_{2}\right)=\frac{1}{2} \frac{\partial}{\partial t}\left\|r_{2}\right\|_{L^{2}(\Omega)}^{2}
$$

then, using the assumption on $A$, we verify that

$$
\left\|\epsilon_{2}\right\|_{L^{2}(\Omega)}^{2}+\frac{1}{2} \frac{\partial}{\partial t}\left\|r_{2}\right\|_{L^{2}(\Omega)}^{2} \leq C\left(\left\|r_{1}\right\|_{L^{2}(\Omega)}^{2}+\left\|r_{2}\right\|_{L^{2}(\Omega)}^{2}+\left\|\epsilon_{1}\right\|_{L^{2}(\Omega)}^{2}\right)
$$

Integrating (3.57) in time and since $r_{2}(T)=0$, applying Gronwall's lemma, we easily obtain the following error estimate:

$$
\left\|\epsilon_{2}\right\|_{L^{2}\left(j ; L^{2}(\Omega)\right)}^{2}+\left\|r_{2}\right\|_{L^{\infty}\left(J ; L^{2}(\Omega)\right)}^{2} \leq C\left\|u-U_{h}\right\|_{L^{2}\left(J ; L^{2}(\Omega)\right)^{2}}^{2}
$$

Then (3.50) follows from (3.58) and the previous statements immediately. 
Collecting Theorems 3.1-3.4, we can derive the following result.

Theorem 3.5 Let (p, $y, \mathbf{q}, z, u)$ and $\left(P_{h}, Y_{h}, Q_{h}, Z_{h}, U_{h}\right)$ be the solutions of (2.5)-(2.11) and (2.38)-(2.44), respectively. Assume that $\left.\left(U_{h}+\widetilde{Z}_{h}\right)\right|_{\tau} \in H^{s}(\tau), \forall \tau \in \mathcal{T}_{h}(s=0,1)$, and that there is $v_{h} \in K_{h}$ such that

$$
\left|\left(U_{h}+\widetilde{Z}_{h}, v_{h}-u\right)\right| \leq C \sum_{\tau \in \mathcal{T}_{h}} h_{\tau}\left\|U_{h}+\widetilde{Z}_{h}\right\|_{H^{s}(\tau)}\left\|u-U_{h}\right\|_{L^{2}(\tau)}^{s}
$$

Then we have that

$$
\begin{gathered}
\left\|u-U_{h}\right\|_{L^{2}\left(j ; L^{2}(\Omega)\right)}^{2}+\left\|y-Y_{h}\right\|_{L^{\infty}\left(j ; L^{2}(\Omega)\right)}^{2}+\left\|\mathbf{p}-P_{h}\right\|_{L^{2}\left(; L^{2}(\Omega)\right)}^{2} \\
+\left\|z-Z_{h}\right\|_{L^{\infty}\left(j ; L^{2}(\Omega)\right)}^{2}+\left\|\mathbf{q}-Q_{h}\right\|_{L^{2}\left(j ; L^{2}(\Omega)\right)}^{2} \leq C \sum_{i=1}^{15} \eta_{i}^{2},
\end{gathered}
$$

where $\eta_{1}$ is defined in Theorem 3.1, $\eta_{2}, \ldots, \eta_{7}$ are defined in Theorem 3.2 , and $\eta_{8}, \ldots, \eta_{15}$ are defined in Theorem 3.3.

\section{Conclusion and future work}

In this paper, we derive new $L^{\infty}\left(L^{2}\right)$ and $L^{2}\left(L^{2}\right)$-posteriori error estimates of the mixed finite element solutions for quadratic optimal control problems governed by semilinear parabolic equations. The a posteriori error estimates for the semilinear parabolic optimal control problems by mixed finite element methods seem to be new.

In our future work, we shall use the mixed finite element method to deal with nonlinear parabolic integro-differential optimal control problems. Furthermore, we shall consider a posteriori error estimates and superconvergence of a mixed finite element solution for nonlinear parabolic integro-differential optimal control problems.

Competing interests

The authors declare that they have no competing interests.

Authors' contributions

All authors contributed equally to the manuscript and read and approved the final manuscript.

\section{Author details}

'School of Mathematics and Statistics, Chongqing Three Gorges University, Chongqing, 404000, P.R. China. ${ }^{2}$ Laboratory for Applied Mathematics, Beijing Computational Science Research Center, Beijing, 100084, P.R. China. ${ }^{3}$ School of Science, Chongqing Jiaotong University, Chongqing, 400074, P.R. China. ${ }^{4}$ Department of Mathematics and Computational Science, Hunan University of Science and Engineering, Yongzhou, 425100, P.R. China.

\section{Acknowledgements}

The authors express their thanks to the referees for their helpful suggestions, which led to improvements of the presentation. This work is supported by the National Science Foundation of China (11201510), Mathematics TianYuan Special Funds of the National Natural Science Foundation of China (11126329), China Postdoctoral Science Foundation funded project (2011M500968), Natural Science Foundation Project of CQ CSTC (cstc2012jjA00003, CSTC, 2010BB8270), Scientific and Technological Research Program of Chongqing Municipal Education Commission (KJ121113, KJ120420), Scientific Research Project of Department of Education of Hunan Province (13C338), and Science and Technology Project of Wanzhou District of Chongqing (2013030050)

Received: 16 July 2013 Accepted: 8 October 2013 Published: 08 Nov 2013

\section{References}

1. Tiba, D: Lectures on the Optimal Control of Elliptic Problems. University of Jyvaskyla Press, Jyvaskyla (1995)

2. Haslinger, J, Neittaanmaki, P: Finite Element Approximation for Optimal Shape Design. Wiley, Chichester (1989)

3. Mcknight, R, Bosarge, W Jr.: The Ritz-Galerkin procedure for parabolic control problems. SIAM J. Control Optim. 11 , 510-524 (1973) 
4. Arada, N, Casas, E, Tröltzsch, F: Error estimates for the numerical approximation of a semilinear elliptic control problem. Comput. Optim. Appl. 23, 201-229 (2002)

5. Chen, Y, Huang, Y, Liu, W, Yan, N: Error estimates and superconvergence of mixed finite element methods for convex optimal control problems. J. Sci. Comput. 42, 382-403 (2009)

6. Chen, Y, Liu, W: A posteriori error estimates for mixed finite element solutions of convex optimal control problems. J. Comput. Appl. Math. 211, 76-89 (2008)

7. Chen, Y, Lu, Z, Huang, Y: Superconvergence of triangular Raviart-Thomas mixed finite element methods for bilinear constrained optimal control problem. Comput. Math. Appl. 66, 1498-1513 (2013)

8. Brunner, $\mathrm{H}$, Yan, N: Finite element methods for optimal control problems governed by integral equations and integro-differential equations. Numer. Math. 101, 1-27 (2005)

9. Liu, W, Yan, N: A posteriori error analysis for convex distributed optimal control problems. Adv. Comput. Math. 15, 285-309 (2001)

10. Liu, W, Yan, N: A posteriori error estimates for optimal control problems governed by Stokes equations. SIAM J. Numer. Anal. 40, 1850-1869 (2003)

11. Hoppe, RHW, Iliash, Y, Iyyunni, C, Sweilam, NH: A posteriori error estimates for adaptive finite element discretizations of boundary control problems. J. Numer. Math. 14, 57-82 (2006)

12. Liu, W, Yan, N: A posteriori error estimates for convex boundary control problems. SIAM J. Numer. Anal. 39, 73-99 (2001)

13. Chen, $Y, L u, Z$ : Error estimates of fully discrete mixed finite element methods for semilinear quadratic parabolic optimal control problems. Comput. Methods Appl. Mech. Eng. 199, 1415-1423 (2010)

14. Chen, Y, Lu, Z: Error estimates for parabolic optimal control problem by fully discrete mixed finite element methods. Finite Elem. Anal. Des. 46, 957-965 (2010)

15. Lu, Z, Chen, Y: A posteriori error estimates of triangular mixed finite element methods for semilinear optimal control problems. Adv. Appl. Math. Mech. 1, 242-256 (2009)

16. Chen, Y, Liu, L, Lu, Z: A posteriori error estimates of mixed methods for parabolic optimal control problems. Numer. Funct. Anal. Optim. 31, 1135-1157 (2010)

17. Liu, W, Yan, N: A posteriori error estimates for optimal control problems governed by parabolic equations. Numer. Math. 93, 497-521 (2003)

18. Lions, JL: Optimal Control of Systems Governed by Partial Differential Equations. Springer, Berlin (1971)

19. Chen, Y, Hou, T: Superconvergence and $L^{\infty}$ error estimates of RT1 mixed methods for semilinear elliptic control problems with an integral constraint. Numer. Math. 3, 432-446 (2012)

20. Babuska, I, Strouboulis, T: The Finite Element Method and Its Reliability. Oxford University Press, Oxford (2001)

21. Carstensen, C: A posteriori error estimate for the mixed finite element method. Math. Comput. 66, 465-476 (1997)

22. Scott, LR, Zhang, S: Finite element interpolation of nonsmooth functions satisfying boundary conditions. Math. Comput. 54, 483-493 (1990)

23. Ciarlet, PG: The Finite Element Method for Elliptic Problems. North-Holland, Amsterdam (1978)

24. Ladyzhenskaya, O, Urlatseva, H: Linear and Quasilinear Elliptic Equations. Academic Press, New York (1968)

25. Eriksson, K, Johnson, C: Adaptive finite elements methods for parabolic problems l: a linear model problem. SIAM J. Numer. Anal. 28, 43-77 (1991)

10.1186/1687-2770-2013-230

Cite this article as: Lu et al.: New a posteriori error estimates of mixed finite element methods for quadratic optimal control problems governed by semilinear parabolic equations with integral constraint. Boundary Value Problems 2013, 2013:230

\section{Submit your manuscript to a SpringerOpen ${ }^{\circ}$ journal and benefit from:}

- Convenient online submission

- Rigorous peer review

- Immediate publication on acceptance

Open access: articles freely available online

- High visibility within the field

- Retaining the copyright to your article 